\title{
Eine vertiefte survey-weltliche Perspektive auf Surveys
}

Die letzten Unterkapitel dienten der Analyse und der Darstellung der verschiedenen Survey-Welten. Das folgende Unterkapitel zielt auf die Vertiefung einzelner Aspekte von survey-weltlichen Fragestellungen, welche durch die empirische Analyse aufgeworfen wurden. Das erste Unterkapitel widmet sich der Dynamik von Survey-Welten. Es sind hierbei insbesondere die Analysen von François Horn zu den Entwicklungsdynamiken von Produktionswelten, welche eine dynamische Perspektive auf die Entwicklung und Transformation von produktionsweltlichen Koordinationsgrundlagen eingeführt haben (Horn 2001). Darauf aufbauend werden produktionsweltliche Dynamiken und Herausforderungen der Survey-Produktion beschrieben und analysiert. Anschließend wird im zweiten Unterkapitel auf die Trennung zwischen Dienstleistungen und Produkten eingegangen, sowie auf die Verbindung zwischen sozialen und marktlichen Strukturen der Beratungswelt (Granovetter 1985). Geklärt wird hier, inwiefern die erbrachten Survey-Dienstleistungen in den verschiedenen Survey-Welten Produkteigenschaften aufweisen und welche Auswirkungen fehlende Produkteigenschaften der hier angebotenen Dienstleistungen auf den Survey-Markt in der Beratungswelt haben. Im dritten Unterkapitel werden die in Abschn. 2.4 dargestellten erweiterten Qualitätskriterien der Prozessperspektive sowohl der öffentlichen Statistik, wie auch der Marktforschung, im Hinblick auf deren survey-weltlicher Bedeutung diskutiert. Zentral ist die Diskussion von unterschiedlichen Relevanzen und Bedeutungen dieser erweiterten Qualitätskriterien aus der Perspektive der verschiedenen Survey-Welten. Im letzten Unterkapitel wird schlussendlich ein neuer Qualitätsrahmen für die Survey-Produktion auf der Basis von Survey-Welten eingeführt. Zentraler Fokus dabei ist die Entstehung methodischer Probleme aus fehlerhaften survey-weltlichen Koordinationen. Damit wird ein konzeptueller Rahmen für die Entstehung 
methodischer Probleme geschaffen, welche auf Koordinationsprobleme in der Survey-Praxis, d. h. insbesondere in der Koordination mit Survey-Firmen, zurückgehen.

\subsection{Die Dynamik von Survey-Welten}

Die zentrale Analyseebene der EC und des Survey-Welten-Ansatzes stellen nicht Organisationen, Institutionen und Produkte dar, sondern deren interpretativen Bezug auf Konventionen und die situative Kompromissschließung zwischen verschiedenen Konventionen. Die genannten Entitäten sind folglich aus Sicht der EC nicht gegeben, sondern werden erst durch den Bezug auf eine Konvention „real“ und interpretierbar (Diaz-Bone 2012; Salais 2003). Deutlich wird durch diese Position jedoch auch, dass Organisationen, Institutionen und Produkte survey-weltlich keine abschließende Handlungslogik beinhalten. Dies wurde ersichtlich in Fall (A). Obwohl (P1) das Survey-Projekt zunächst eher als dienstleistungsweltlich fundiert auffasste, wobei er sich maßgeblich auf das Objekt „Pretest" stützte, nahm das Survey-Projekt im Verlauf der Verhandlungen zwischen dem Auftraggeber und der Survey-Firma einen stärker beratungsweltlichen Charakter an (vgl. Abschn. 6.1.7). Klar wird damit, dass sich weder die Organisation, noch das durch sie verkaufte „Produkt“ survey-weltlich fixieren lässt, sondern dass alleine die bei der Projektbearbeitung zur Koordination hinzugezogenen Konventionen über die effektive survey-weltliche Grundlegung entscheiden. In diesem Kapitel werden im empirischen Material identifizierte typische survey-weltliche Transformationen untersucht. Obwohl diese keine Vollständigkeit beanspruchen können, dienen sie der Illustration von Mechanismen survey-weltlicher Transformationen.

Eine klassische Transformation von der Beratungswelt ausgehend stellt die „Produktentwicklung“ dar. Eine Produktentwicklung findet dann statt, wenn in der Beratungswelt typische Kundenbedürfnisse identifiziert werden, auf deren Basis in der Folge ein standardisiertes Angebot erstellt wird. Dies findet typischerweise in Survey-Firmen statt, welche sowohl in der Beratungs- wie auch in der Dienstleistungswelt tätig sind. So kann beispielsweise bei der Beratung von Klienten bei organisationalen Problemen wiederholt festgestellt werden, dass eine Mitarbeitenden-Zufriedenheitsbefragung sinnvoll für das Steuern der Tätigkeiten des Bereiches des Human Ressource-Managements auch bei kleineren Firmen ist. Diese wiederholte Konfrontation mit der Angemessenheit von Mitarbeitenden-Befragungen auch in kleineren Firmen kann nun der Auslöser dafür 
sein, ein umfassendes Produkt zu entwickeln, d. h. ein standardisiertes Vorgehen, Anwendungsbereiche, Ziele etc. zu definieren. Dieses kann dann als Survey-Produkt der Firma angeboten werden. Durch die Standardisierung wird das Vorgehen in der Folge nicht mehr wie bisher anhand des zu untersuchenden Falles entwickelt, sondern basiert auf den verschiedenen, zu Beginn der Produktentwicklung definierten Parametern.

Eine umgekehrte Transformation von der Dienstleistungswelt hin zur Beratungswelt findet dann statt, wenn in größeren Kundenorganisationen interne Marktforschungsabteilungen infolge von Rationalisierungsmaßnahmen geschlossen werden. Dadurch fehlt eine interne Stelle, welche eine Übersetzungsleistung zwischen den Bedürfnissen der internen Klienten und den Angeboten der Survey-Firmen leistet (vgl. für die potenziell daraus entstehenden methodischen Probleme Abschn. 7.4). Die Übersetzung einer Problemlage in eine Fragestellung und Methodik findet in diesem Fall nicht mehr in Zusammenarbeit mit einer internen Marktforschungsstelle statt, sondern muss künftig direkt in Zusammenarbeit mit der beauftragten Survey-Firma stattfinden. Durch den Wegfall der Übersetzungsleistung der internen Marktforschungsabteilung findet dadurch eine Änderung des survey-weltlichen Koordinationsrahmens statt. Denn vor der Schließung der internen Marktforschungsabteilungen konnten diese die Skalenvorteile der Dienstleistungswelt nutzen, was für die internen Klienten nach der Schließung nicht mehr möglich ist, da sie nicht über die entsprechende Kompetenz in Survey-Fragen verfügen.

Eine durch Survey-Firmen oft genannte Veränderung von Kundenbedürfnissen stellt die Normierung von Erhebungsinstrumenten dar. Gemeint ist hier eine Vereinheitlichung des Erhebungsprozesses, welche durch international tätige Firmen aus Gründen der Vergleichbarkeit der verschiedenen Erhebungen und damit der Kommunikationsfähigkeit gefordert wird. Diese Entwicklung von standardisierten Produkten hin zu einer uniformen statistischen Form wird im folgenden Zitat durch einen langjährigen Projektleiter einer Survey-Firma beschrieben. Hierbei beantwortet (B1) die Frage nach unterschiedlichen Kundensegmenten der verschiedenen Survey-Firmen in der Schweiz: ${ }^{1}$

${ }^{1}$ Die durch (B1) beschriebene Entwicklung der Standardisierung stellt aus der Perspektive des Survey-Welten-Ansatzes eine Uniformierung dar, da die Standardisierung über verschiedene Survey-Firmen hinweg stattfindet. Dies geht zugleich mit einem Verlust der für die Dienstleistungswelt charakteristischen Flexibilität bei der Bearbeitung von Kundenwünschen einher. 
B1: Oh, das ist schwierig zu sagen, also unser Eindruck ist, dass die internationalen Konzerne auf internationaler Konzernebenen, beim internationalen Konzern [International tätige Survey-Firma] fragen die Produkte, die wollen wir einsetzen. Und die werden dann in allen Ländern eingesetzt. [...] Das wird überall das Gleiche, also durch [...]. Die haben dann einfach Verträge auf globaler Ebene [...]. Die haben Accounts-Teams auf globaler Ebene. Und und, dann weiß einfach die die Zentrale, die Konzernleitung es wird in der Schweiz gleich gemacht wie in Mexiko. Also können wir die Zahlen, also die Ergebnisse einigermaßen, ja, vergleichbar interpretieren. Das ist so der Hintergrund. Geht dann auch ums Reporting. Wenn es natürlich in jedem Land wieder anders geforscht wird. Kann man es gar nicht für ein zentrales Reporting brauchen.

In diesem Zitat macht (B1) deutlich, dass durch die Globalisierung von internationalen Firmen auch eine Nachfrage nach international standardisierten Erhebungsmethoden bestünde, welche zu einer Uniformierung der SurveyProduktion führt. Die bisherige Standardisierung der Produkte durch (einzelne) Survey-Firmen reicht folglich nicht mehr aus, da die Standardisierung neu transorganisational und damit über verschiedene Erhebungen hinweg uniformiert ausfallen muss. Dies stellt eine Transformation des Kundenbedürfnisses von der Dienstleistungs- zur Informationswelt dar, da neu eine generische Qualität gefordert wird. Die standardisierten Angebote einzelner Survey-Firmen müssen international gleichgeschaltet werden, um eine Vergleichbarkeit der einzelnen Erhebungen ermöglichen zu können. Diese Uniformierung führt dazu, dass eine statistische Form entsteht, auf welche sich unterschiedliche Survey-Firmen oder Niederlassungen einer Survey-Firma beziehen können, wenn sie die uniformierten Parameter der statistischen Form einhalten. Eine Möglichkeit dazu besteht in der Vergabe von Lizenzen für Survey-Firmen. Ein Beispiel hierfür stellen die Sinus-Milieus dar, welche vom Sinus-Institut an andere Survey-Firmen lizenziert werden (SINUS Markt- und Sozialforschung GmbH 2017; M.I.S Trend S.A). Daraus folgt jedoch, dass eine zentrale Eigenschaft der Dienstleistungswelt, nämlich die Flexibilität im Eingehen auf die Kundenwünsche, verloren geht. Denn obwohl in der Dienstleistungswelt ein standardisiertes Marktangebot herrscht, findet doch ein Eingehen auf individuelle Kundenwünsche statt, d. h. das Angebot kann an die spezifischen Wünsche angepasst werden. Diese Möglichkeit besteht jedoch im Zuge der Transformation hin zur Uniformierung, d. h. der Erstellung einer statistischen Form, nicht mehr.

Die Entwicklung von statistischen Formen der Informationswelt wurde bereits erwähnt (vgl. Abschn. 5.4). Hier findet eine Transformation der Entdeckungen der akademischen Welt hin zu einem Informationsinstrument statt. Die zum Teil nur schwierig zu vergleichenden und potenziell auch 
widersprüchlichen Forschungsergebnisse und Prozeduren werden durch die Informationswelt $\mathrm{zu}$ einheitlichen Forschungskonzepten und Indikatoren verdichtet. Jedoch existiert auch eine umgekehrte Dynamik der Entwicklung von der Informationswelt hin zur akademischen Welt, welche als „Krise von statistischen Formen" bezeichnet werden kann. In Abschn. 5.4 wurde die Problematik der Informationswelt rekonstruiert, welche aus den vielfältigen Ansätzen und Theorien der akademischen Welt möglichst eindeutige, allgemein geltende Indikatoren für surveybasierte Informationsprojekte entwickeln muss. Als Beispiel wurde hierfür das Thema der Wohlbefindensforschung in der Sozialberichterstattung gewählt. Die Problematik bestand hierbei für die Autoren darin, sich zwischen den verschiedenen existierenden Theorien der Wohlbefindensforschung für einen Ansatz zu entscheiden, bzw. einen Kompromiss zu etablieren. Denn im Gegensatz zur akademischen Welt ist es für die Informationswelt nicht möglich, eine Pluralität der Ansätze zu verfolgen, wie dies in der akademischen Welt der Fall ist (vgl. Abschn. 5.4). Wie weiter dargestellt wurde, stellt die akademische Welt eine wichtige Grundlage für die Informationswelt dar. Denn hier wird Forschung zu Konzepten und Indikatoren betrieben, welche als Grundlage für die Entwicklung von statistischen Formen dienen. Diese Transformation von akademischen Konzepten und Indikatoren in Richtung Informationswelt kann jedoch auch in umgekehrter Richtung erfolgen. Dies geschieht dann, wenn informationsweltliche Konzepte und Indikatoren in die Krise geraten, d. h. ihr Realitätsgehalt von einem breiten Publikum oder auch von Akademikern angezweifelt wird. Eine solche Krise der Informationsproduktion führt dazu, dass eine erneute Auseinandersetzung mit den akademischen Diskursen gesucht werden muss, um die allgemeine Akzeptanz von Informationen wieder herstellen zu können. Ein Beispiel für eine solche „Krise“ stellt die Kritik des capabilities-Ansatzes an Indikatoren dar, welche lediglich die den Personen zur Verfügung stehenden Mittel als Maßstab für deren Wohlbefinden nehmen (Salais und Villeneuve 2009, S. 6 ff.). ${ }^{2}$ Aus Sicht des capability-Ansatzes reicht dies jedoch nicht aus, da die Verfügung über Mittel noch nicht bedeutet, dass diese auch effektiv und gemäß den individuellen Wünschen genutzt werden können. Aus Sicht des capabilities-Ansatzes sind folglich Indikatoren notwendig, welche fähig sind, nebst den Ressourcen zusätzlich auch die daraus entstehenden Möglichkeiten abzubilden. Obwohl diese Kritik des capabilities-Ansatzes keine

\footnotetext{
${ }^{2}$ Vgl. für eine ausführliche Darstellung dieses Ansatzes Amartya Sen (2011). Für einen Überblick zur Kritik von Indikatoren aus der Perspektive der EC auch Diaz-Bone (2018, S. $357 \mathrm{ff}$.).
} 


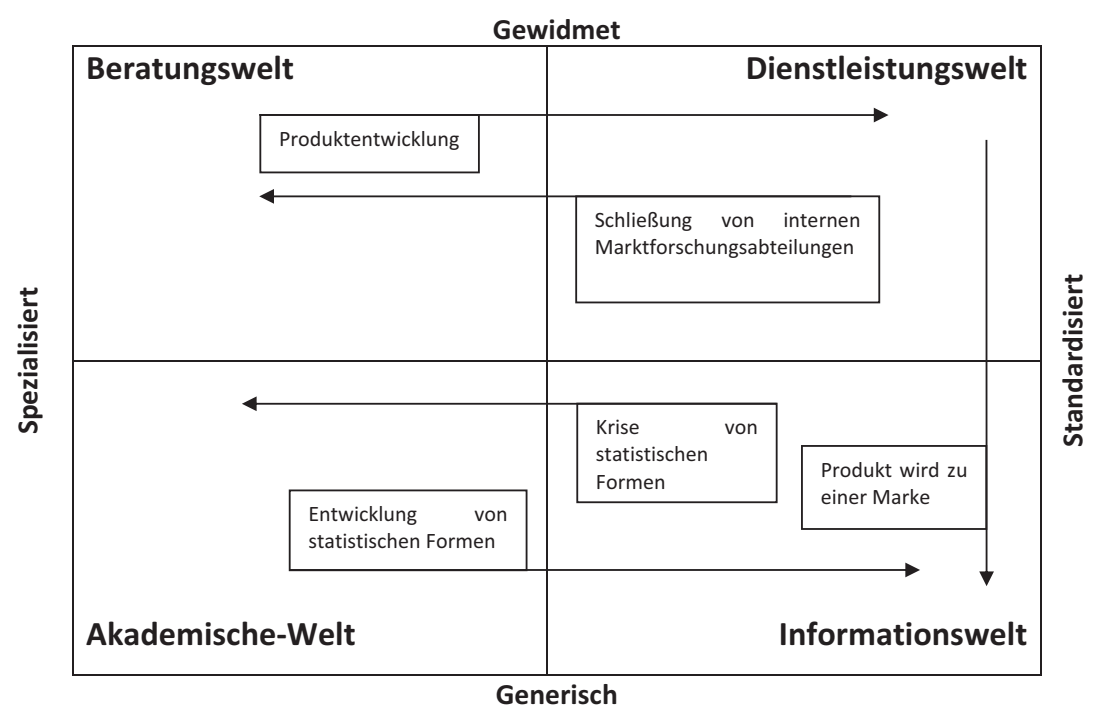

Abb. 7.1 Die Dynamik von Survey-Welten

tatsächliche Krise der ressourcenorientierten Indikatoren hervorzurufen imstande ist, so wird dennoch der Mechanismus der Respezifizierung von eigentlich „objektiven“ Indikatoren der Informationswelt durch deren Bezug auf Diskussionen der akademischen Welt deutlich. ${ }^{3}$ Würde die vom capabilities-Ansatz artikulierte Kritik breite Zustimmung zukommen, so hätte dies einen Bruch der „Objektivität“ und der Akzeptanz von ressourcenorientierten Indikatoren zur Folge. Die Krise von Indikatoren basiert folglich auf einer Infragestellung des Objektivitätsanspruch der in dieser Welt verwendeten Indikatoren und Konzepten und macht eine Wiederherstellung von Objektivität durch den Bezug auf Theorien und Diskurse der akademischen Welt notwendig. Die verschiedenen Dynamiken zwischen Survey-Welten sind in Abb. 7.1 zusammengefasst dargestellt.

${ }^{3}$ Die Kritik an der scheinbaren Objektivität von Indikatoren der Informationswelt stellt die Stoßrichtung vieler ,politischer“ Arbeiten von Forschenden der EC, insbesondere von Robert Salais, dar. Vgl. hierzu Salais (2006, 2008a, 2010). 
In diesem Kapitel wurde die Dynamik von Survey-Welten thematisiert. Wie dargestellt wurde, lassen sich die „Produkte“ der verschiedenen Survey-Welten nicht unbedingt über die Zeit stabil der gleichen Survey-Welt zuordnen. Vielmehr können diese Transformationen und Dynamiken unterworfen sein. Zudem wurde nachgezeichnet, wie Welten untereinander dependent sein können. Eine klassische Austauschbeziehung wurde hier zwischen der Informationswelt und der akademischen Welt identifiziert. Die akademische Welt stellt dabei eine zentrale Basis für die Entwicklungen von Konzepten und Indikatoren für die Informationswelt dar. Die Transformation von Erkenntnissen der akademischen Welt hin zu Informationen, bzw. Konzepten der akademischen Welt zu Indikatoren der Informationswelt, ist nicht ohne Übersetzungsverluste möglich. Denn im Gegensatz zur akademischen Welt muss sich die Informationswelt auf einheitliche Konzepte und Indikatoren festlegen. Survey-weltliche Dynamiken können aber auch unintendiert ablaufen. Ein Beispiel hierfür stellen Wachstumsabsichten von Survey-Firmen dar, welche hauptsächlich in der Beratungswelt tätig sind. Ein Wachstum birgt die Gefahr, dass Arbeitsprozesse durch Economies of Scale arbeitsteiliger werden, was in der Folge zu einer veränderten Produktontologie führt. So findet eine survey-weltliche Entwicklung einer Firma statt, ohne dass dies zwingend mit Absicht geschieht. Deutlich wird damit die enge Kopplung zwischen der Koordinationsform und der produzierten SurveyQualität. ${ }^{4}$

\subsection{Der Produktcharakter von Surveys und die Funktionsweise von Survey-Märkten}

In Abschn. 3.3.2 wurde das Konzept der Global-Value-Chains eingeführt. Dabei wurde darauf hingewiesen, dass diese Theorie eine diachrone Perspektive in die Analyse von Survey-Welten einführt. Survey-Welten stellen aus diesem Blickwinkel folglich nicht mehr lediglich eine Kombination von Konventionen dar. Unterschiedliche Konventionen lassen sich dadurch vielmehr zusätzlich auf verschiedene Stationen der Produktionskette beziehen. Dies zeigt sich besonders deutlich in der (empirischen) Analyse von tatsächlichen Produktionswelten, da hier Kompromisse und Konflikte ebenfalls in konkreten Situationen etabliert

\footnotetext{
${ }^{4}$ Hier könnte folglich von einer Gleichschaltung von „Justice“ und „Justesse“ im Hinblick auf die Produktqualität gesprochen werden (Boltanski und Thévenot 2007, S. 184).
} 
werden und so unterschiedlichen Stationen im Produktionsprozess zugeordnet werden können. Bei der Darstellung der Rezeption der Global-Value-Chain-Forschung durch die EC wurde jedoch auf einen zentralen Kritikpunkt durch die EC verwiesen. Der Beitrag der Global-Value-Chain-Forschung zum Verständnis des Funktionierens von Märkten besteht darin, dass die Reduktion von ökonomischen Austauschprozessen auf „Markt“ und „Hierarchie“, bzw. „Organisation" der Transaktionskostenökonomie aufgebrochen wird und hybride Settings zwischen diesen beiden Polen aufgezeigt werden (Sturgeon 2009). ${ }^{5}$ Zugleich werden aber neoklassische Elemente der Marktkonzeption auch in der Global-Value-Chain-Forschung weiter verwendet. So wird die Qualität von Produkten nicht weiter problematisiert und als gegeben erachtet (Diaz-Bone 2013). Direkt damit einher geht ein Festhalten am rationalen Akteur. Denn durch die feststehende Qualität von Produkten ist die Produktbewertung für Marktakteure unproblematisch und sie können folglich als rationale Akteure Marktvergleiche und -abwägungen vornehmen. In den folgenden Absätzen wird eine Kritik an dieser verallgemeinernden Marktkonzeption aus der Perspektive der Survey-Welten vorgenommen. ${ }^{6}$ Kritisiert wird die Annahme einer objektiven Produktqualität. Aufgezeigt wird hier am Beispiel der Beratungswelt, inwiefern hier nicht von einer objektiven Produktqualität gesprochen werden kann und was dies für den „Markt“ in dieser Welt bedeutet.

In der Darstellung der verschiedenen Survey-Welten wurde versucht, die Spezifität des Produkts in jeder Survey-Welt herauszuarbeiten. Wie bereits im Kapitel zur Beratungswelt dargestellt, besteht hier in seiner idealtypischen Form dieser Survey-Welt das Problem, dass sich kein wirkliches Angebot auf Seiten der Survey-Firma findet und dass beim Kauf der Beratung unklar ist, wie genau das eingekaufte Produkt aussehen wird. Deutlich wird hier ein dem neoklassischen Marktkonzept konträr entgegenstehendes Funktionieren des Marktes. Durch das Fehlen eines von außen sichtbaren, objektiven Marktangebotes sind es in dieser Welt viel stärker soziale Strukturen und Einbettungen, welche eine effiziente Koordination zwischen Klient und Survey-Firma ermöglichen (Granovetter

\footnotetext{
${ }^{5}$ Vgl. für die Perspektive der Transaktionskostenökonomie Coase (1937) und Williamson (1987).

${ }^{6}$ Die empirische Grundlage der folgenden Ausführungen stellen wie bereits bei der Darstellung der Survey-Welten einerseits die Interviews mit verschiedenen Survey-Praktikern und andererseits die teilnehmenden Beobachtungen bei verschiedenen Survey-Firmen dar.
} 


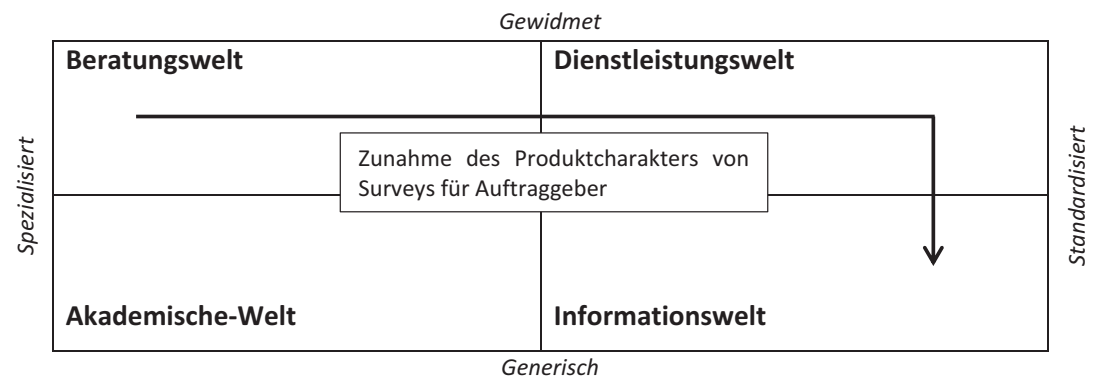

Abb. 7.2 Der Produktcharakter von Surveys aus der Perspektive der Endkunden

1985). ${ }^{7}$ In der Dienstleistungswelt findet infolge der Standardisierung bereits eine stärkere Kodifizierung des Marktes statt. Kunden können hier anhand einiger marktüblicher Qualitätsindikatoren, aber auch durch den Vergleich mit ähnlichen Survey-Projekten die Qualität des eingekauften Surveys bereits besser abschätzen. ${ }^{8}$ Am stärksten weisen jedoch die in der Informationswelt verkauften Surveys einen Produktcharakter auf. Surveys sind in dieser Welt aus Sicht des Endkunden durch eine Vergleichbarkeit gekennzeichnet und damit am stärksten mit anderen Surveys vergleichbar. Der größte Unterschied liegt jedoch darin, dass die Befragungen zum Zeitpunkt des Einkaufs eines Surveys bereits durchgeführt wurden. So kaufen Kunden der WEMF-Studien nicht Befragungen ein, sondern existierende surveybasierte Daten zu einem festen Preis (WEMF AG für Werbemedienforschung 2017). Da die Erhebung bereits stattgefunden hat, liegen bereits konkrete Daten zu den Eigenheiten der Befragung vor. Insgesamt kann so

\footnotetext{
${ }^{7}$ Und folglich könnte man mit Mark Granovetter von einer Welt der Embeddedness sprechen, da in dieser Welt der Rückgriff auf die soziale Einbettung besonders stark und unumgänglich ist für das Funktionieren des wirtschaftlichen Austauschs in der Beratungswelt (Granovetter 1985).

${ }^{8}$ Callon et al. haben aufgezeigt, wie in Märkten versucht wird, Konkurrenz zu umgehen. Ein zentrales Diktum ist dabei, dass auf dem Markt angebotene Produkte einerseits vergleichbar sein müssen, um überhaupt als Produkt wahrgenommen werden zu können. Andererseits müssen Produkte voneinander unterschiedlich sein, um direkte Konkurrenz und somit Ersetzbarkeit umgehen zu können (2002). Bezogen auf die Dienstleistungswelt wird damit deutlich, dass auch dort nicht ein einheitliches, standardisiertes Angebot über die verschiedenen Survey-Firmen hinweg existiert, da sich die Angebote der verschiedenen Survey-Firmen unterscheiden müssen, um so allzu direkte Konkurrenz vermeiden zu können, aber dass dennoch Gemeinsam- und Vergleichbarkeiten existieren.
} 
eine Zunahme der Eigenschaft von Surveys von der Beratungs- zur Dienstleistungs- und weiter zur Informationswelt festgestellt werden. In Abb. 7.2 wird die aufgezeigte Entwicklung des Produktcharakters für Endkunden über verschiedene Survey-Welten hinweg dargestellt:

$\mathrm{Zu}$ beachten ist, dass dies die Perspektive der Endkunden darstellt. Im Gegensatz zur Welt der Beratung und der Dienstleistung sind die Welt der Information und die akademische Survey-Welt elementar dreiteilige Value-Chains (vgl. hierzu Abschn. 5.4 und 5.5). Bei der akademischen Survey-Welt macht die Diskussion um Produkt-Charakteristika für „Endkunden“ keinen Sinn, da hier kein privatwirtschaftlicher Verkauf von Daten stattfindet. ${ }^{9}$ In der Informationswelt sind, wie bereits erwähnt, die Produkteigenschaften von Surveys am stärksten in ihrer privatwirtschaftlichen Ausrichtung. Anders stellt sich dieses Problem jedoch aus der Perspektive der Kundenorganisation dar. Infolge der industriellen Auftragsweitergabe zwischen Survey-Firma und Kundenorganisation weisen Surveys hier keine vorfixierten Eigenschaften als Produkt auf (vgl. Abschn. 5.4). Denn die Produktion von Surveys orientiert sich gerade nicht am Angebot von SurveyFirmen, sondern direkt an den Aufträgen der Auftraggeber.

Die Erklärung der Global-Value-Chain-Forschung für die von ihr (analytisch) herausgearbeiteten Koordinationsformen liegt in unterschiedlichen, objektiv feststellbaren Eigenschaften von Gütern. Angenommen werden folglich zwei Dinge: Einerseits objektiv feststellbare Eigenschaften von Gütern ${ }^{10}$ und andererseits ideale Koordinationsformen, welche sich graduell zwischen den Polen „Markt“ und „Hierarchie" (in Form einer integrierten Organisation) bewegen (Gereffi et al. 2005: S. $73 \mathrm{ff}$.). Insbesondere mit Blick auf die Beratungswelt wird jedoch deutlich, dass Surveys (als Güter) nicht objektive Eigenschaften aufweisen, welche dann zu einer idealen Koordinationsform führen. Denn es sind nicht Surveys selber, welche objektiv - das heißt über verschiedene Survey-Welten hinweg unterschiedliche Eigenschaften aufweisen, sondern es sind die unterschiedlichen survey-weltlichen-Konventionen, welche den Surveys ihre spezifische wissensontologische Qualität verleihen. Bezogen auf die GVC-Forschung wird folglich deutlich, dass es nicht „objektive“ Qualitäten der Güter sind, welche unterschiedliche Koordinationsformen hervorrufen, sondern die die Produktqualität organisierenden Konventionen (Daviron und Ponte 2005, S. 204 ff.).

\footnotetext{
${ }^{9}$ Diese Diagnose trifft sich mit derjenigen von François Horn. Auch dieser rechnet den südwestlichen Quadranten der Produktionswelten der staatlichen und open-source Software-Produktion zu (Horn 2004, S. 18).

${ }^{10} \mathrm{Zu}$ einer Kritik und Analyse vgl. Diaz-Bone (2013, S. 436 f.).
} 
Die von der Neoklassik, wie auch der Global-Value-Chain-Forschung angenommenen Elemente von Märkten wie der rationale Akteur und die objektive Qualität von Gütern, können folglich nicht grundsätzlich vorausgesetzt werden. ${ }^{11}$ Dies wird besonders deutlich mit Blick auf die Beratungswelt. Wie argumentiert wird, fehlen dafür zwei grundlegende Elemente: Zunächst ist es gerade die Eigenheit der Beratungswelt, dass Klienten die Lösung für Probleme und nicht Antworten auf fertig formulierte Forschungsfragen suchen. Dies impliziert einerseits, dass die Klienten in dieser Welt nicht über das inhaltliche Wissen für eine Problemlösung verfügen. Es impliziert aber auch, dass sie nicht über die für die Survey-Forschung notwendigen methodischen Kompetenzen verfügen. ${ }^{12}$ Dadurch fehlt in diesem wirtschaftlichen Austausch - wie in den letzten Absätzen dargestellt - die für einen neoklassisch anonymen Markt notwendige objektive Produktqualität. Durch die in dieser Welt durch Surveys zu lösenden spezifischen Probleme zeigt sich die eigentliche Qualität der Dienstleistung - in deutlicher Absetzung zur Informationswelt - erst durch das Erbringen derselben. Die Spezifität der Problemstellung verhindert folglich im Vornhinein einen Abgleich mit ähnlichen Fällen. Der „Markt“ der Beratungswelt stellt deswegen ein besonders anschauliches Beispiel für eine Ökonomie des Einzigartigen dar, wie sie Lucien Karpik beschreibt (2011). Das Konzept des standardisierten (und damit objektiven) Marktes verortet er dabei über die Neoklassik hinaus in einer Art Grundkonsens der Sozialwissenschaften:

Das anthropologische Modell, auf dem der Gegensatz von Markt und Kultur beruht, hat eine lange Geschichte und lebt auch in den heutigen Sozialwissenschaften weiter. Seine binäre Struktur verleiht ihm seine Radikalität: In der Kultur bleibt das Einmalige erhalten, im Markt geht es verloren (Karpik 2011: S. 16).

\footnotetext{
${ }^{11}$ Karpik kritisiert ironisch die neoklassische Konzeption von Märkten und die damit zusammenhängende Annahme einer objektiven Produktqualität, welche ihm gemäß erst durch eine ominöse Substanz ermöglicht wird: „In der neoklassischen Theorie kann der Austausch nur existieren, weil es eine Substanz mit ungewöhnlichen Eigenschaften gibt: Sie macht sofort alles sichtbar, was ihr begegnet, unentgeltlich und an jedem beliebigen Ort. Sie begründet eine einheitliche Welt für die gesamte Menschheit" (Karpik 2011, S. 51).

${ }^{12}$ Sichtbar wird dadurch die Notwendigkeit einer ,prozeduralen Rationalität“, anstelle einer „substanziellen Rationalität“ als Bewertungsgrundlage von Marktteilnehmenden (Favereau 1989a, S. 280). „Rationalität“ wird aus der Perspektive der prozeduralen Rationalität nicht als anthropologische Konstante betrachtet, sondern als Ergebnis des Bezugs von Akteuren auf situative Elemente und Konventionen.
} 
Karpik weist jedoch darauf hin, dass eine Vielzahl an Marktformen existiert, auf welchen nicht-standardisierte und somit nicht-vergleichbare Produkte gehandelt werden. ${ }^{13}$ Als Beispiel nennt er den Markt der Psychoanalyse. Im Gegensatz zum anonymen neoklassischen Marktmodell seien hier persönliche Beziehungen nicht die Ausnahme, sondern im Gegenteil gerade die Regel (Karpik 2011, S. 28). Deutlich wird damit, dass sich die Qualität der erbrachten Dienstleistung erst während der Dienstleistung zeigt. Eine objektive Abschätzung und Wahl der Psychoanalyse gemäß dem neoklassischen Marktmodell ist folglich gar nicht möglich. Bei der Lösung dieser Herausforderung sieht Karpik jedoch soziale Strukturen am Werk, welche die Qualitätseinschätzung auch von zunächst unvergleichbaren Produkten zu erhöhen helfen. Zentral sind für Karpik hier sog. Koordinationsinstanzen. Er differenziert dabei zwischen sechs unterschiedlichen Instanzen: Persönlichen Netzwerken, Praktikernetzwerken, Kennzeichnungen, Ratgebern, Bewertungen und Kanalisierungen (Karpik 2011, S. 62 f.).

Wie bereits dargestellt, kann die Beratungswelt als eine Form der Ökonomie des Einzigartigen verstanden werden. Denn auch hier wird das eigentlich eingekaufte „Produkt“ erst sichtbar, wenn es bereits produziert ist. Diese Survey-Welt hat jedoch nicht nur das Problem der Kommunikation der hier hergestellten Survey-Qualität. Das Problem liegt tiefer, da die produzierte Survey-Qualität zunächst gar keine Interessenten hat. Wie in der Darstellung zur Beratungswelt beschrieben wurde (vgl. Abschn. 5.2), ist es gerade die Eigenheit dieser Welt, kein vermarktbares „Angebot" ${ }^{“ 14}$ zu erstellen, an welchem sich potenzielle Kunden orientieren und mit ihren individuellen Wünschen abgleichen können. Vielmehr werden hier proaktiv Probleme in Firmen aufgenommen und durch die Survey-Firma auf Basis von Survey-Umfragen gelöst. Der Unterschied zwischen Beratungs- und Dienstleistungswelt liegt entsprechend in der Artikulationsfähigkeit von Problem- und Fragestellungen der Klienten, bzw. Kunden. Dies beinhaltet zwei Punkte: Einerseits die Fähigkeit, das vorliegende organisationale und durch Surveys zu lösende Problem konzeptuell fassen zu können. Andererseits die methodische Fähigkeit der Transformation dieses Problems in ein survey-basiertes Forschungsdesign. Durch diesen doppelten Kompetenzmangel besteht eine große Asymmetrie, das heißt eine große kognitive Ambiguität

\footnotetext{
${ }^{13}$ Und er fragt deswegen: „Warum soll das Besondere nicht Teil des Marktes sein?“ (Karpik 2011, S. 17 f.).

${ }^{14}$ Bereits im Angebots-Begriff steckt die Idee der dienstleistungsweltlichen Koordination. Durch dieses standardisierte Angebot hindurch können Akteure ihre individuellen Wünsche in standardisierte Wünsche artikulieren. Vgl. hierzu Storper und Salais (1997, S. 20).
} 
zwischen Berater und Klient (Centemeri 2012, S. 21). Es ist diese kognitive Ambiguität, welche dazu führt, dass Klienten in dieser Welt die Qualität der Dienstleistung nicht ohne weiteres einschätzen können, was das in Abschn. 5.2 beschriebene Vertrauensverhältnis notwendig macht. Im folgenden Interviewausschnitt macht dies der interviewte langjährige Projektleiter aus der Beratungswelt deutlich, nachdem er zunächst den großen Vorteil dieser Survey-Welt - das individuelle Eingehen auf die Probleme des Klienten - hervorgehoben hat. Nachdem er darauf hingewiesen hat, dass seine Firma Befragungen nicht mehr selber durchführt, sondern an größere Survey-Firmen mit einer umfassenden Befragungsinfrastruktur auslagere, macht er folgende Aussage:

[...] wir schauen nur, dass der Kunde das richtige, die richtige Qualität bekommt, aber unter uns gesagt, ist das auch nicht ähm, das Erfolgsrezept, weil der Kunde bezahlt natürlich mehr bei uns, weil wir mehr Beratung machen und kann die Qualität gar nicht richtig einschätzen. Das kommt dann dazu.

Der langjährige Projektleiter macht zunächst deutlich, dass seine Firma nur für das Design der Befragung zuständig sei, jedoch nicht für die Befragung selbst, welche ausgelagert wird. Er macht aber deutlich, dass dies nicht unbedingt den einzigen Weg zum Erfolg darstelle, da der Preis infolge dieser Produktionsorganisation entsprechend (im Vergleich zur Dienstleistungswelt) höher sei. Zusätzlich könnten Klienten die spezifische Qualität dieser Art der SurveyProduktion nicht einschätzen. Sichtbar wird in diesem Zitat das doppelte Marktproblem der Beratungswelt. Einerseits fehlen die für einen neoklassischen, anonymen Markt notwendigen rationalen Akteure, da den Klienten in der Beratungswelt das dafür notwendige Wissen fehlt. ${ }^{15}$ Außerdem sind die Dienstleistungen dieser Welt aufgrund deren Spezifität und dadurch der Unmöglichkeit der Nutzung von Skaleneffekten, auch durch einen höheren Preis im Vergleich zur Dienstleistungs- oder Informationswelt, gekennzeichnet. Genau dies wird im vorhergehenden Zitat ausgedrückt und es wird dadurch deutlich, dass die Beratungswelt auch für den Interviewpartner nicht ohne weiteres als Königsweg der Survey-Produktion gilt, sondern als Nische, welche entsprechende Vor- und

\footnotetext{
${ }^{15}$ Damit wird nicht gesagt, dass die Märkte in der Dienstleistungs- und der Informationswelt als anonyme, neoklassische Märkte funktionieren. Die Abweichung vom rationalen Akteur ist jedoch in der Beratungswelt besonders hoch, deswegen wird diese Welt hier als Beispiel herangezogen.
} 
Nachteile (auch aus Sicht der Survey-Firma) mit sich bringt, die beachtet werden müssen.

Durch die fehlende Artikulationsfähigkeit aufseiten der Klienten existiert in der Beratungswelt in einem engeren Sinne kein anonymer Markt, in welchem individuelle Wünsche mit bestehenden Angeboten abgeglichen und in der Folge dasjenige Angebot mit der besten Übereinstimmung zwischen beiden Seiten gewählt werden kann. Potenzielle Kunden in der idealtypischen Beratungswelt können folglich selber gar nicht in Kategorien der Survey-Forschung denken, sodass man paradox formulieren könnte, dass die idealtypische Ausprägung dieses Bereichs des Survey-,,Marktes“ ohne einen eigentlichen Markt, das heißt ohne ein sichtbares Marktangebot, auskommen muss. ${ }^{16}$ Denn wie im Kapitel zur Beratungswelt dargestellt wurde, zeigt sich die eigentliche Qualität des „Produkts" erst in der direkten Beziehung zwischen dem Berater und dem Klienten. Dies wird auch im folgenden Zitat eines langjährigen Projektleiters deutlich:

Die [Anm. RV: Die Klienten] wissen das nicht. Die können die Qualität auch nicht richtig abschätzen. Das läuft nur bei wirklich guten Beziehungen. Und ich ich mach wirklich von zehn Anfragen, die ich hab, mach ich neun Mal den Auftrag. Das gelingt mir dann doch. Bei [vorherigem Arbeitgeber] war vielleicht jeder dritte was, oder? Dreißig Prozent. Also, oder? Aber wir sind zu wenig bekannt. Das ist extrem anstrengend und ich hole viele Aufträge halt über die [Nebentätigkeit] [...].

Der Projektleiter verweist in diesem Zitat zunächst auf die fehlende Kompetenz der Klienten, die Qualität der Beratungswelt in einem anonymen, standardisierten Markt abschätzen zu können. Für die Qualitätsvermittlung seien funktionierende persönliche Beziehungen notwendig, was dazu führe, dass die Hauptschwierigkeit in dieser Welt die Kontaktaufnahme zum Klienten sei. Käme der Kontakt jedoch zustande, so sei die Wahrscheinlichkeit einer Zusammenarbeit sehr hoch. Ein Problem sieht der Projektleiter dabei auch in der geringen Bekanntheit seiner Survey-Firma. Dies hat den Effekt, dass viele der von ihm behandelten Survey-Projekte über Kontakte durch die Zweittätigkeit der Survey-Firma zustande kommen. Hier wird einerseits die bereits angesprochene Problematik der fehlenden Methodenkompetenz aufseiten des Klienten sichtbar. Es wird aber auch die Ambivalenz der von dem Interviewten verfolgten Geschäftsstrategie erkennbar. Einerseits weist er auf die hohe Erfolgsquote des Verhältnisses zwischen

\footnotetext{
${ }^{16}$ Dies ist der Grund, warum Survey-Projekte der gewidmeten Welten oft einen Kompromiss zwischen Beratungs- und Dienstleistungswelt darstellen.
} 
Anfragen und Aufträgen hin, andererseits wird klar, dass die Zahl der Anfragen im Vergleich zum vorherigen Arbeitgeber geringer und zudem andere Strategien der Klientengewinnung notwendig sind. Dies bezeichnet er als „extrem anstrengend“" verweist aber zugleich auf eine Strategie der Klientengewinnung. Diese besteht darin, Klienten über eine Nebentätigkeit der Firma zu gewinnen. Dadurch, dass sich die Qualität dieser Welt erst durch den persönlichen Kontakt zeigt, stellt sich die bereits existierende Nebentätigkeit als ideale Gelegenheit dar, neue Klienten anzuwerben. Denn dadurch kann der spezifische Nutzen einer Zusammenarbeit im direkten Kontakt aufgezeigt werden. Erkennbar wird an dieser Aussage, wie die wirtschaftliche Koordination des Zusammenfindens von Survey-Firma und Klient und die Qualitätskonstruktion der hier verkauften Produkte trotz des Fehlens eines neoklassischen anonymen Marktes infolge einer direkten Embeddedness in bestehenden sozialen Strukturen geschieht (Granovetter 1985).

Die im Vergleich zu den anderen Survey-Welten „späte“ Sichtbarwerdung der Survey-Qualität macht dabei eine spezifische Verkaufsstrategie notwendig. In den Ausführungen zur Beratungswelt wurde diese als „foot in the door" bezeichnet (vgl. Abschn. 5.2). Wie bereits im vorangehenden Zitat deutlich wird, besteht eine zentrale Kompetenz von Projektleitenden in dieser Welt darin, überhaupt in Kontakt mit potenziellen Klienten zu treten. Denn dies ist die notwendige Voraussetzung dafür, dass die spezifische Qualität dem Klienten aufgezeigt werden kann. Erst dann wird die inhaltliche und methodische Kompetenz des Beraters auf die Probleme des Klienten bezogen und so der Mehrwert - und damit auch die spezifische Qualität - einer survey-basierten Erhebung für den Klienten einsehbar. Im folgenden Zitat nimmt der Interviewpartner, welcher bereits in verschiedenen Survey-Welten tätig war, eine Spezifizierung der Konsequenzen dieser Qualitätsentwicklung für Projektleitende der Beratungswelt vor:

Gestern habe ich den Bodensee und den Genfersee gesehen, das hat auch noch nicht mancher gesehen [...]. Aber es hat sich gelohnt, zumindest in Genf, also in Lausanne, das hat sicher sich gelohnt, da muss man einfach flexibel sein, und die, sie müssen sehen, viele in der Marktforschung, die können nicht verkaufen, die wollen im Büro sitzen und ihre Studien vom Kunden erhalten und abwickeln. Und abends um 5 und 6 gehen, vielleicht gibt es noch die fleißigen, die dann noch masochistisch bis 8 Uhr arbeiten, aber die gehen nicht raus, ich gehe Türklinken putzen, ich war gestern bei einer Versicherung [...] Da müssen sie halt einfach hin, aber das können viele nicht.

Der Projektleiter erwähnt in diesem Zitat zunächst seine intensive Reisetätigkeit und verweist darauf, dass dies nicht der Regelfall in der Marktforschung sei. Er moniert folglich, dass es hier einen Unterschied gebe zur Arbeitsweise 
eines Großteils der Marktforschung, bei welcher Projektleitende auf Auftragserteilungen warten. Im Gegensatz dazu gehe er aktiv auf potenzielle Klienten/ Kunden zu, was jedoch auch spezifische Kompetenzen voraussetze. Da die Qualität erst im Kontakt sichtbar wird, gilt es, die Qualität zum Kunden zu bringen. Deutlich wird hier, dass der Transport der spezifischen Qualität zum Klienten eine Kernaufgabe in dieser Welt darstellt. Diesem Heranbringen an den Klienten kommt folglich in der alltäglichen Arbeit in der Beratungswelt ein entsprechend hoher Stellenwert zu. In Absetzung zu anderen Projektleitenden kann in dieser Welt nicht auf einen Auftrag gewartet werden, welcher infolge eines breit sichtbaren Marktangebots als Anfrage eines Kunden beginnt. Eine zentrale Aufgabe für Projektleitende in dieser Welt besteht folglich darin, Kontakte herzustellen. Der Stellenwert dieser Tätigkeit wird im Zitat deutlich, da die die beruflich bedingte Mobilität (das Reisen) von der Bodenseeregion zur Genferseeregion wohl den ganzen Tag ausgefüllt hat und es wird eine Survey-Welten-spezifische Investition der Zeit ersichtlich. Im vorhergehenden Zitat wird aber auch erkennbar, dass ein einst hergestellter Kontakt mit einer hohen Erfolgswahrscheinlichkeit im Hinblick auf eine Zusammenarbeit belohnt wird. Festgehalten werden kann folglich, dass trotz der paradoxen Ausgangslage dieses Marktes ohne eigentlichen „Markt“ eine Vermittlung zwischen Survey-Firma und Klient hergestellt werden kann. Diese findet jedoch nicht durch ein sichtbares und standardisiertes Angebot statt, wie dies idealisiert in der neoklassischen Theorie des Marktes gedacht ist. In seiner idealtypischen Form findet dies jedoch auch nicht über ein spezialisiertes Angebot statt, das heißt durch grobe Methodenkategorien, welche dann spezifisch auf die Wünsche und Bedürfnisse ${ }^{17}$ des Kunden zugeschnitten werden, wie dies in der Dienstleistungswelt der Fall ist. ${ }^{18}$ In der Beratungswelt

\footnotetext{
${ }^{17}$ Bereits in dieser Zweiteilung von „Bedürfnissen“ und „Wünschen“ zeigt sich der Kompromisscharakter zwischen zwei Survey-Welten. „Bedürfnisse“ weist gerade auf die fehlende Artikulationsmöglichkeiten im Hinblick auf eine Auswahl in einem Markt hin, während ein Wunsch diese Artikulationsfähigkeit voraussetzt.

${ }^{18}$ Diese Eigenheit des Survey-Marktes in der Dienstleistungswelt ist gut in der folgenden Aussage eines erfahrenen Survey-Praktikers zu sehen: „Das sind dann eben Standardprodukte, ja. Und das gibts bei uns nicht. Also wir haben so Ideen auf der Homepage, wo man sehen kann, ok, wie kann man Preisforschung machen, wie kann man Markenforschung machen. Aber dann wir hören immer mal zuerst dem Kunden zu, was er braucht und haben dann ein maßgeschneidertes Angebot, wo wir halt unsere Fragebogen dann zusammenstellen auf Grund von Fragen, die wir kennen, die funktionieren, die hier jetzt auch passen sollten. Aber das wird immer, äh, maßgeschneidert, neu kreiert dann einzeln. [...] Das sind einfach wie wir Roh, ähm ich sag wie bei einem Handwerker so gewisse
} 
sind es vielmehr persönliche Beziehungen und Kontakte, welche das Zustandekommen von marktlichen Austauschbeziehungen ermöglichen. Deutlich wird folglich die umfassende soziale Einbettung des wirtschaftlichen Handelns in dieser Welt (Granovetter 1985). In den Daten konnten fünf Modelle identifiziert werden, anhand derer das Marktproblem der Beratungswelt aufgelöst wird: ${ }^{19}$

1. Wiederholte Zusammenarbeit: Durch eine erste Zusammenarbeit entsteht eine vertrauensbasierte Zusammenarbeit auf Zeit. ${ }^{20}$ Die mit den Surveys betraute projektleitende Person wird so zur Ansprechpartnerin für Survey-Fragen. ${ }^{21}$ Sobald sich Probleme in der eigenen Organisation zeigen, wird ein Berater nach Lösungsmöglichkeiten für das Problem angefragt. Die Ausarbeitung der Fragestellung und der Problemlösungsvorschläge geschieht dann, wie in der Beratungswelt beschrieben.

2. Vermittlung durch Drittparteien: Surveys sind mittlerweile eine weit verbreitete Methode, um organisationale Probleme zu lösen. Surveys sind als Möglichkeiten der Wissensproduktion auch bei Werbe- und Marketingagenturen, bei Beratungsfirmen etc. etabliert. Durch Austauschbeziehungen zwischen Survey-Firmen und diesen Intermediären kann sich die Empfehlung für ein datenbasiertes Vorgehen ergeben. ${ }^{22}$ Hier ist zwischen zwei Vorgehensweisen zu unterscheiden. Einerseits kann der Intermediär eigenmächtig einen

Rohteile hat, der hat noch [...]. So Reste schleifen muss, ja, die die, die die rohen Stücke haben wir schon, die wir da aus der Schublade nehmen können. Aber es ist nicht schon einfach fix-fertig“. Deutlich wird hier jedoch auch, dass hier bereits ein Kompromiss mit der Beratungswelt geschlossen wird, da hier ,maßgeschneidert“ auf die Situation des Kunden/ Klienten eingegangen wird.

${ }^{19}$ Die folgenden Ausführungen basieren einerseits auf Interviews mit Mitarbeitenden von Survey-Firmen, andererseits auf den ethnographischen Beobachtungen bei Survey-Firmen.

${ }^{20} \mathrm{Wie}$ bereits argumentiert, zeigt sich die eigentliche Qualität des hier erstellten Produktes erst im direkten Kontakt. Dieser Kontakt ist stark personengebunden. Wechseln folglich die Mitarbeitenden in einer Organisation, muss auch die Kundenbeziehung neu aufgebaut werden.

${ }^{21}$ Zentral ist hier die Abgrenzung gegenüber wiederholten Zusammenarbeiten, welche durch eine eingespielte Koordination begründet ist (d. h. genaugenommen in der guten Übereinstimmung zwischen individuellen Wünschen und dem Angebot der Survey-Firma). Diese sind der Dienstleistungswelt zuzurechnen.

${ }^{22}$ Die genannten weiteren Organisationen, welche als Vermittler fungieren, stellen hier Intermediäre dar (Bessy und Chauvin 2013). Obwohl sie scheinbar Aufträge „,nur“ weitergeben, findet hier dennoch eine Formatierung des Auftrags statt. 
Survey-Auftrag formulieren und an eine Survey-Firma weitergeben. In diesem Fall stellt Koordination einen Kompromiss mit der Dienstleistungswelt dar, da hier eine standardisierte Auftragsvergabe stattfindet. Die zweite Option besteht darin, dass sich ein Joint-Venture ergibt, in welchem die Kundenorganisation, Intermediär und Survey-Firma gemeinsam an der Lösung eines organisationalen Problems arbeiten.

3. Kombination von Arbeitsfeldern: Nicht alle Organisationen, welche Marktund Sozialforschung durchführen, konzentrieren sich ausschließlich auf dieses Arbeitsfeld. Möglich sind Kombinationen mit weiteren Arbeitsfeldern wie der Marketingberatung, Webapplikationen, der Medienanalyse etc. Kooperationen in einem anderen Bereich als der Markt- und Sozialforschung können dazu führen, dass auf die Möglichkeiten einer survey-basierten Problemlösung hingewiesen wird. ${ }^{23}$ In diesem Fall findet also eine Queraquirierung von Surveyaufträgen statt.

4. Akquise aus der Dienstleistungswelt: Eine Möglichkeit, die Qualität der Beratungswelt zu vermitteln, bilden Offertanfragen. ${ }^{24}$ Hierbei werden Preise für eine bestimmte Dienstleistung erfragt. Die Grundidee entspricht hier der Dienstleistungswelt, da individuelle Wünsche erfüllt werden sollen. Hier kann jedoch eine Transformation der Koordinationsgrundlagen stattfinden, indem Projektleitende durch das Aufzeigen alternativer Forschungsdesigns einen Beratungsansatz in der Offertbeantwortung vornehmen. Auch bei dieser Form der Beziehungsherstellung findet ein Kompromiss zwischen der Beratungsund der Dienstleistungswelt statt, welcher ja nach Koordinationsform nach der Kontaktaufnahme in seinen Welten-Anteilen schwanken kann.

\footnotetext{
${ }^{23}$ Dies lässt sich anhand folgender Aussage gut nachvollziehen, in welchem ein langjähriger Survey-Forscher auf die Problematik der Tätigkeit in der Beratungswelt und zugleich seine Strategie zur Überwindung von deren Markt-Problem aufzeigt: „Aber wir sind zu wenig bekannt. Das ist extrem anstrengend und ich hole viele Aufträge halt über die [Zweite Firmentätigkeit], weil ich dort auch im Verkauf tätig bin und in der Beratung. Dann switcht das rüber. Das läuft dann so, also die sagen dann, wir haben auch noch Mafo [Anm. RV: Kurzform für Marktforschung], was ihr macht Mafo? Könnt ihr das? Hab ich gesagt, ja selbstverständlich". Hier wird folglich die Strategie der Querakquirierung aus anderen Geschäftsfeldern deutlich.

${ }^{24}$ Ersichtlich wird hier, dass dies eine hybride Organisation im Hinblick auf die surveyweltliche Ausrichtung bedingt. Die entsprechende Survey-Firma muss folglich in beiden Welten tätig sein und je nach Projekt einen unterschiedlichen survey-weltlichen Schwerpunkt festlegen.
} 
5. Persönliche Beziehungen: Generell sind gute persönliche Beziehungen zentral in der Beratungswelt. Es bietet sich folglich an, auf bereits bestehende persönliche Beziehungen auch im geschäftlichen Austausch zurückzugreifen. Marktbasierte Austauschbeziehungen können so auf bereits bestehende persönliche Beziehungen aufbauen (Bekannte, Verwandte, ehemalige Arbeitskollegen etc.). Zentral sind dabei nicht unbedingt nur feste persönliche Beziehungen, sondern insbesondere auch ,weak ties“ (Granovetter 1973).

In den verschiedenen Möglichkeiten zeigt sich, wie ein Markt ohne „Markt“ zustande kommt, bzw. wie wirtschaftliche Austauschbeziehungen jenseits eines standardisierten Marktes zustande kommen können. Mit einem eigentlichen Marktparadox ist man jedoch auch bei der Betrachtung der Informationswelt konfrontiert. Denn das hier angestrebte Wissensformat der Information konkurriert mit der Idee eines offenen, durch Wettbewerb gesteuerten Marktes. Im Kapitel zur Informationswelt wurde dargelegt, dass die Produktion von Informationen auf statistischen Formen mit einer hohen Reichweite aufbaut. Denn erst die Vereinheitlichung der Survey-Produktion ermöglicht die Produktion vergleichbarer Informationen. Zentral ist dabei nebst der Vereinheitlichung des Erhebungs- und Auswertungsprozesses insbesondere die Vereinheitlichung der verwendeten Kategorien. Damit ist einerseits eine Vereinheitlichung über die Zeit gemeint, andererseits eben auch eine Vereinheitlichung über verschiedene Erhebungsorganisationen, bzw. Erhebungsprojekte, hinweg. Diese Vereinheitlichung steht nun jedoch in Konkurrenz mit der Idee eines durch Wettbewerb getriebenen Marktes, auf welchem verschiedene Anbieter mit unterschiedlichen, aber dennoch vergleichbaren Produkten um die Gunst des Kunden konkurrieren (Callon et al. 2002; Godart und White Harrison C. 2007). Denn die Idee dieses „Marktes" besteht gerade darin, für wirtschaftliche oder soziale Felder - welche globale Ausdehnungen einnehmen können - für alle in diesen Feldern tätigen Akteuren relevante Informationen zu produzieren. Der Markt für Informationen ist folglich gar nicht an einem eigentlichen „Markt“ ausgerichtet, da dieser das spezifische Wissensformat dieser Welt konkurrieren würde. Im Gegensatz zur Beratungswelt existiert hier jedoch gerade nicht eine Ökonomie des Singulären (Karpik 2011), sondern man könnte hier von einer „Ökonomie des Totalen“ sprechen. In Absetzung zur neoklassischen Theorie des Marktes könnte man folglich paradox formulieren, dass in dieser Welt ein funktionierender Markt besteht, wenn Monopole den Markt dominieren, sei dies durch staatliche oder privatwirtschaftliche Organisationen.

Deutlich wurde in den vorhergehenden Ausführungen die unterschiedliche Funktionsweise von „Märkten“ in den verschiedenen Survey-Welten. Es wurde 
sichtbar, dass sich die durch die GVC-Forschung und auf der Theorie der Transaktionskostenökonomie basierende Aufteilung von Märkten zwischen den Polen „Markt" und „Organisation“ lediglich auf die standardisierten Welten übertragen lässt (Gereffi et al. 2005). In der Welt der Survey-Beratung fehlt für beide Organisationsformen schlicht eine der wirtschaftlichen Transaktion vorhergehende Definition der Qualität von Surveys. Dadurch wird deutlich, dass aus der Perspektive der Survey-Welten der von der GVC-Forschung angenommene Markt - wie auch immer im Detail ausgestaltet - selbst das Ergebnis eines umfassenden Konstruktionsprozesses darstellt. Denn der von der Beratungs-, zur Dienstleistungs- und weiter zur Informationswelt zunehmende Produktcharakter von Surveys ergibt sich nicht lediglich durch eine unterschiedliche Marktform, sondern basiert auf der zunehmenden Investition in Marktformen (Thévenot 1984). Die Objektivität des Marktes in der Dienstleistungs- und der Informationswelt basiert folglich nicht auf rationalen Akteuren im Sinne der neoklassischen Wirtschaftstheorie, sondern auf kollektiven Investitionen in Marktformen, durch die eine Rationalität erst möglich wird. ${ }^{25}$ Fehlen diese kollektiven Investitionen, wie dies in der Beratungswelt der Fall ist, so müssen andere Wege gefunden werden, um dennoch einen „Markt“ etablieren zu können. Insgesamt wird dadurch die problematische Annahme einer objektiv feststellbaren Qualität von Produkten in der GVC-Forschung deutlich. Wie dargestellt, lassen sich jedoch auch beim formal gleichen Produkt „Survey“ unterschiedliche Qualitäten identifizieren. Aus der Perspektive der EC ist es der Bezug auf unterschiedliche (Produktions-)Konventionen, welche diese unterschiedlichen Qualitäten erst ermöglicht (Storper und Salais 1997).

\subsection{Eine Kritik von Qualitätskriterien der Survey- Methodologie}

Wie in Kap. 2 dargelegt wurde, stellt die Definition von über die klassische Qualitätstrias hinausgehenden Qualitätskriterien und der Miteinbezug der Anwendersituation bei der Nutzung von statistischen Daten den maßgeblichen Beitrag der

\footnotetext{
${ }^{25}$ Eine umfassende Charakterisierung von Marktobjektivität hat André Orléan vorgenommen. Die Annahme der Existenz einer Marktobjektivität basiert gemäß ihm auf vier Postulaten: Der Existenz eines Sets an Gütern und Qualitäten, welches allen Marktteilnehmenden bekannt ist, gemeinsamen Annahmen über die Zukunft, der kollektiven Akzeptanz eines zentralen Preismechanismus und der Einnahme einer strikt utilitaristischen Perspektive auf Rohstoffe.
} 
Prozessperspektive dar (Eurostat 2000; Statistics Canada 2009). Prominente Beitragende für die Qualitätsdiskussion der Survey-Methodologie waren Biemer und Lyberg (2008). ${ }^{26}$ Gleichzeitig wurde in Kap. 2 dargelegt, dass die Prozessperspektive zwar einerseits über die Datenperspektive auf Survey-Qualität hinausgeht, indem sie die Nutzungssituation von Survey-Daten miteinbezieht, in welcher Survey-Qualität produziert wird. Zugleich wurde jedoch argumentiert, dass sie nicht in der Lage ist, die unterschiedlichen Qualitäts- und Koordinationslogiken der verschiedenen Survey-Welten als zusätzliche Qualitätsdimensionen von Survey-Qualität zu berücksichtigen. Die Grundlage der folgenden Diskussion bildet deswegen die Erkenntnis, dass Qualitätskriterien nicht unabhängig von den unterschiedlichen in Kap. 5 dargestellten Qualitätslogiken der verschiedenen Survey-Welten sind. Wie in der folgenden Diskussion auch gezeigt wird, lassen sich Qualitätskriterien darüber hinaus auch nicht von den unterschiedlichen Koordinationsformen der statistischen Kette der verschiedenen Survey-Welten trennen.

In den folgenden Absätzen werden die erweiterten Qualitätskriterien der Prozessperspektive auf dieser Grundlage kritisch auf ihr Verhältnis für survey-weltliche Qualitäts- und Koordinationsfragen hin diskutiert:

\section{Relevanz (Relevance of statistical concepts)}

The statistical concepts used for statistics are relevant if they meets [sic!] users' needs. The identification of users and their expectations is therefore necessary (Eurostat 2000, S. 2).

\footnotetext{
${ }^{26}$ Eine Diskussion dieser Qualitätskriterien wird ebenfalls durch Desrosières vorgenommen (2009a). Allerdings bezieht sich Desrosières nicht auf die unterschiedliche Bedeutung und Relevanz dieser Kriterien für verschiedene Survey-Welten, sondern auf die unterschiedliche Bedeutung und Relevanz dieser Kriterien für einerseits inhaltliche Fachspezialisten und andererseits methodisch tätige Statistiker bei der Erstellung von Statistiken. Die Ausgangslage dieser Diskussion stellt die Feststellung durch Desrosières dar, wonach Statistiken zugleich ,real“ und „konventionenbasiert“ sind. Diese bezieht er auf die spezifische Arbeitsteilung während des Produktionsprozesses von Statistiken, bei welcher die zweite Gruppe der Statistiker „real“ misst, was die erste Gruppe als „konventionelle“ Kategorien vorgibt (ebd.: S. 311). Die Feststellung von Desrosières, wonach die Qualität von statistischen Daten nicht beurteilt werden kann ohne den Miteinbezug der Spannung zwischen den „realen“ und den „konventionenbasierten“ Aspekten (Desrosières 2000, S. 185), stellt in der folgenden Diskussion der Qualitätskriterien der Prozessperspektive die Ausgangslage dar, da die survey-weltliche Diskussion der erweiterten Qualitätskriterien eine konstruktivistische Perspektive auf Statistiken voraussetzt. Denn erst durch einen konstruktivistischen Standpunkt werden unterschiedliche Formationsregeln (Foucault 2008, S. 504 ff.) von Kategorien in den verschiedenen Survey-Welten plausibel.
} 
Im Hinblick auf die Relevanz als Kriterium für die statistische Produktion zeigt sich ein deutlicher Unterschied zwischen den gewidmeten und den generischen Welten (Storper und Salais 1997, S. 29 ff.). Denn wie in den entsprechenden Kapiteln zu den verschiedenen Survey-Welten deutlich wurde, unterscheiden sich diese insbesondere im Hinblick auf eine Eigenschaft der statistischen Produktion. So richten sich die in generischen Welten produzierten Daten wie bereits bei Storper und Salais beschrieben - nicht an einzelnen Statistikkonsumenten aus, sondern am anonymen Kollektiv der Statistikkonsumenten. Es ist diese generische Orientierung, welche für den Umstand verantwortlich ist, dass insbesondere in der Informationswelt die Statistikkonsumenten nicht auch die Kunden der Survey-Firma darstellen und die generischen Welten oft durch eine grundlegend dreiteilige statistische Kette gekennzeichnet sind (vgl. Abschn. 5.4). Das Kriterium der Relevanz bekommt dadurch in der Informationswelt seine spezifische Bedeutung, da hier Daten produziert werden, bevor deren spezifische Anwendung feststeht. Sichergestellt werden soll mit dem Kriterium der Relevanz, dass die produzierten Daten bei der Nutzung der Daten auch tatsächlich einen Informationswert besitzen. Im Gegensatz dazu ist das Kriterium der Relevanz von Daten für die Beratungswelt kein sinnvolles Kriterium, da die Daten in dieser Welt lediglich für den Berater relevant sein müssen. Für Klienten können in dieser Welt Daten gar nicht relevant sein, da die Klienten kaum fähig sind, die Daten selber zu interpretieren. Relevanz als Kriterium bezieht sich in dieser Welt folglich nicht auf Daten wie in der Informationswelt, sondern auf Empfehlungen. In der Dienstleistungswelt kommt dem Kriterium der Relevanz eine mittlere Relevanz zu, da hier einerseits die auftraggebende Organisation zugleich auch die datennutzende Organisation ist. In dieser Welt findet jedoch oft eine Vermittlung der Datenproduktion durch interne Marktforschungsstellen statt, sodass bei der Übersetzung von Interessen in Forschungsfragen und bei der anschließenden Übermittlung des Auftrags an die Survey-Firma durchaus Fragen der Relevanz für die schlussendlichen Statistikkonsumenten eine Rolle spielen können. In der akademischen Survey-Welt bekommt Relevanz als Kriterium für die Survey-Forschung wiederum eine eigene Bedeutung. Denn die Relevanz wird hier nicht durch einzelne Statistikkonsumenten entschieden, sondern durch den Bezug der Resultate auf den akademischen Diskurs. Relevanz ist dadurch weniger ein Kriterium im Hinblick auf die situative Nutzung von Daten wie in der Informationswelt, sondern für das Potenzial der Generierung neuartiger Erkenntnisse für den akademischen Diskurs. 
Genauigkeit (Accuracy of estimates)

Accuracy is the difference between the estimate and the true parameter value. Assessing the accuracy is not always possible, due to financial and methodological constraints (Eurostat 2000, S. 2).

Das Kriterium der Genauigkeit ist für sämtliche Survey-Welten relevant. Denn schlussendlich sind sämtliche Akteure, welche mit Surveys zum Zweck der Wissensgenerierung arbeiten, darauf angewiesen, dass die Daten auch die zu messenden Eigenschaften möglichst genau abbilden. Jedoch zeigen sich im Hinblick auf dieses Qualitätskriterium Unterschiede in Bezug auf das der Messung zugrunde liegende Messsystem der verschiedenen Survey-Welten. So müssen Daten beispielsweise in der Beratungswelt ein Maß an Genauigkeit beinhalten, welche es für den Berater ermöglicht, Empfehlungen zu treffen. Der relevante Bezug für die Genauigkeit stellt in der Dienstleistungswelt hingegen die auftraggebende Organisation dar. Genauigkeit muss hier in dem Maß erreicht werden, wie sie einen Unterschied für die Kundenorganisation macht. Durch das Installieren von Survey-Erhebungen als konstantem Wissenslieferanten in der Informationswelt ist es in dieser Survey-Welt ein grundlegender Anspruch an die Genauigkeit der Daten, dass diese einen verlässlichen Vergleich zwischen den verschiedenen Erhebungen und dabei insbesondere eine Einschätzung von Trends und Veränderungen ermöglichen. In der akademischen Welt dagegen ist ein $\mathrm{Ma}$ an Genauigkeit notwendig, welche das Widerlegen von etabliertem Wissen ermöglicht.

Zeitlichkeit (Timeliness and punctuality in disseminating results)

Most users want up-to-date figures, which are published frequently and on time at pre-established dates (Eurostat 2000, S. 2).

Das Kriterium der Zeitlichkeit wird von Eurostat als regelmäßige Publikation von Daten zu angekündigten Terminen verstanden. Der informationsweltliche Einfluss dieses Qualitätskriteriums ist damit unschwer erkennbar, da die Informationswelt die einzige Survey-Welt darstellt, in welcher regelmäßig zu angekündigten Zeitpunkten Daten publiziert werden. In Bezug auf die verschiedenen Survey-Welten werden unterschiedliche Bedeutungen dieses Qualitätskriteriums sichtbar. In der Beratungswelt ist die Zeitlichkeit durch die zeitgemäße Abwicklung des Beratungs-Auftrages vorgegeben. Innerhalb dieses 
Zeitbudgets muss sich die beratende Organisation jedoch selber um eine funktionierende zeitliche Organisation kümmern. In der Dienstleistungswelt dagegen ist die zeitliche Lieferung on-time organisiert, d. h. zentral ist die Lieferung der Daten zum vereinbarten Zeitpunkt. Die Informationswelt dagegen ist an staatliche und betriebswirtschaftliche Zeitlogiken gebunden, wie beispielsweise Jahreszahlen, Quartalszahlen etc., wie dies auch in dem durch Eurostat definierten Qualitätskriterium der Zeitlichkeit zum Ausdruck kommt. Die Zeitlichkeit der Erhebung in der akademischen Welt richtet sich an den Entwicklungen des Fachgebietes aus. Zeitlichkeit ist hier insofern sekundär, als dass hier eine verspätete Erhebung kaum Abstriche in der Relevanz der Daten mit sich bringt.

Zugänglichkeit und Klarheit der Information (Accessibility and clarity of the information)

Statistical data have most value when they are easily accessible by users, are available in the forms users desire and are adequately documented. Assistance in using and interpreting the statistics should also be forthcoming from the providers (Eurostat 2000, S. 2).

Die Zugänglichkeit der Daten ist für die Beratungswelt kein sinnvolles Kriterium. Denn hier ist es der datenerhebende Berater selbst, für den die Zugänglichkeit der Daten gewährleistet sein muss, da dem Klienten in dieser Welt lediglich datenbasierte Empfehlungen übermittelt werden. Klarheit der Information ist für diese Welt ebenfalls kein sinnvolles Qualitätskriterium, da in dieser Welt gerade aus Gründen der Verständlichkeit und Interpretierbarkeit keine Daten, sondern Empfehlungen übermittelt werden. In der Dienstleistungswelt besitzt das Kriterium der Zugänglichkeit eine mittlere Relevanz. Denn einerseits ist die Auftraggeber- in dieser Welt auch die Nutzerorganisation der Daten. Andererseits kann die Auftragsvergabe in dieser Welt durch firmeninterne Stellen vermittelt sein, wie etwa durch Marktforschungsabteilungen, sodass die Zugänglichkeit zu den Daten zu einem sinnvollen Qualitätskriterium für diese Welt werden kann. In der Informationswelt kommt diesem Kriterium ein hoher Stellenwert zu, da durch die grundlegend dreiteilige statistische Kette eine erhöhte Distanz zwischen Datenproduzenten und Datenkonsumenten durch die notwendige Vermittlung eines Datenintermediärs (Bessy und Chauvin 2013) wie beispielsweise Bundesämtern, Branchenorganisationen, NGO etc. besteht. ${ }^{27}$ Zugänglichkeit bekommt hier eine spezifische Bedeutung und kann als Öffentlichkeitsprinzip von Daten beschrieben

\footnotetext{
${ }^{27}$ Vgl. zum Intermediär-Konzept in der EC Bessy und Chauvin (2013).
} 
werden. Zugänglichkeit ist in dieser Welt überdies kritisch, da die Statistikkonsumenten in dieser Welt nicht über Spezialkenntnisse im Hinblick auf den Umgang mit Daten verfügen. Dadurch werden verschiedene Maßnahmen notwendig, wie beispielsweise die Nutzung von Datenformaten, welche von der interessierten Öffentlichkeit mit einer durchschnittlichen Ausstattung (insbesondere Software) genutzt werden können. Diese Notwendigkeit der „Assistenz“ gegenüber dem Datenkonsumenten wird auch in der Beschreibung durch Eurostat reflektiert. Zugänglichkeit als Qualitätskriterium bezieht sich in der akademischen Welt hingegen nicht auf den Datenzugang von Statistikkonsumenten, sondern auf den Zugang zu Daten durch die scientific community zwecks der Überprüfung der durch Forschende verbreiteten Erkenntnisse.

\section{Interpretierbarkeit (Interpretability)}

To make appropriate use of statistical information from the NSO [Anm. RV: National Statistical Office] clients have to know what they have and to understand the properties of the information. That requires the NSO to provide descriptions of the underlying concepts, variables and classifications that have been used, the methods of collection, processing and estimation used in producing the information, and its own assessment of the accuracy of the information. We will refer to this property of statistical information as its interpretability (Brackstone 1999, S. 4).

Das Kriterium der Interpretierbarkeit wird von Brackstone in Anlehnung an das Quality-Movement der öffentlichen Statistik definiert und ist mit dem Kriterium der Zugänglichkeit und Klarheit eng verbunden. Der Unterschied besteht jedoch darin, dass bei diesem Kriterium ein stärkerer Fokus auf die Kompetenzen der Statistiknutzenden gelegt wird. Unterschiede im Hinblick auf die Interpretierbarkeit von Ergebnissen gehen mit der Achse spezialisiert - standardisiert einher. Die Dienstleistungs- wie auch die Informationswelt richten sich an ein breites Publikum. Insbesondere die Informationswelt ist auf eine allgemeine Interpretierbarkeit angewiesen, um eine hohe raumzeitliche Reichweite der statistischen Formen erreichen zu können, welche zentral für den Informationswert des in dieser Welt produzierten Wissens ist. Die Dienstleistungswelt richtet sich zwar an ein weniger breites (Kunden-) Publikum und zusätzlich müssen hier Kunden über Survey-Know-how verfügen. Dennoch müssen die hier produzierten Daten für ein breites Kundensegment interpretierbar sein. Die Interpretierbarkeit muss folglich auf die survey-methodologischen Kenntnisse und Fähigkeiten der Kunden zugeschnitten sein. Die Interpretierbarkeit stellt dagegen in der Beratungswelt kein sinnvolles Qualitätskriterium dar, da die Daten lediglich für die beratende Person interpretierbar sein müssen. Denn diese übersetzt die erhobenen Daten in 
Empfehlungen für die Klienten. Für die Klienten selbst bekommt das Kriterium der Interpretierbarkeit eine eigene Bedeutung, da damit eher die Bezugsmöglichkeit der Empfehlungen auf die zu lösenden organisationalen Probleme gemeint ist, was als „Relevanz der Empfehlungen“ übersetzt werden kann. Auch in der akademischen Welt spielt das Kriterium der Interpretierbarkeit eine sekundäre Rolle, da die Aussagen der Daten ohnehin an ein Expertenpublikum gerichtet ist. Die Fähigkeit, diese Daten interpretieren zu können stellt ein Kriterium dafür dar, überhaupt in die Diskussionen um die erhobenen Daten eingebunden zu sein. Interpretierbarkeit bekommt auch in dieser Welt eine eigene Bedeutung, da hier die Interpretierbarkeit eher die Nachvollziehbarkeit von Effekten und Kausalitäten durch die scientific community bezeichnet.

\title{
Vergleichbarkeit (Comparability)
}

\begin{abstract}
Statistics for a given characteristic have the greatest usefulness when they enable reliable comparisons of values taken by the characteristic across space and over time. The comparability component stresses the comparison of same statistics between countries in order to evaluate the meaning of aggregated statistics at the European level (Eurostat 2000, S. 2).
\end{abstract}

In der Beratungswelt stellt die Vergleichbarkeit kein sinnvolles Qualitätskriterium dar. Denn das individuelle Eingehen auf die Problemsituation des Kunden stellt sich hier einer Vergleichbarkeit einerseits mit Erhebungen von anderen Organisationen, andererseits mit früheren Erhebungen derselben Survey-Firma, entgegen. Denn das Ziel einer Erhebung in dieser Welt zielt gerade auf die Lösung eines individuellen Problems. Die Vergleichbarkeit von Daten in der Dienstleistungswelt ist durch die standardisierte Datenerhebung zumindest für Erhebungen durch dieselbe Survey-Firma gegeben, falls dies durch den Kunden nachgefragt wird. Die Vergleichbarkeit mit Erhebungen von anderen Survey-Firmen, bzw. mit unterschiedlichen Erhebungsinstrumenten, ist dabei der Kundenorganisation überlassen und nicht das primäre Ziel der Dienstleistungswelt. Die Vergleichbarkeit der Daten in Bezug auf Zeit und Raum, welche durch einheitliche statistische Formen begründet wird, stellt ein zentrales Qualitätskriterium dieser Welt dar. Denn es ist die Vergleichbarkeit zwischen verschiedenen Erhebungen (räumlich und/oder zeitlich voneinander getrennt), welche den Informationswert von Erhebungen ausmacht. In der akademischen Survey-Welt schließlich stellt die Vergleichbarkeit ein zweischneidiges Schwert dar. Daten müssen einerseits neue Aussagen, das heißt nur bedingt vergleichbare Aussagen zu früheren Erhebungen 
ermöglichen. Andererseits wird dieser Wissensgewinn erst durch den Bezug auf etablierte Theorien und Wissenskonzepte möglich, wodurch in dieser Welt eine Gratwanderung zwischen der Erarbeitung neuartiger Konzepte und deren Bezug auf etabliertes Wissen unternommen werden muss.

\title{
Kohärenz (Coherence)
}

\begin{abstract}
When originating from a single source, statistics are coherent in that elementary concepts can be combined reliably in more complex ways. When originating from different sources, and in particular from statistical surveys of different frequencies, statistics are coherent insofar as they are based on common definitions, classifications and methodological standards. The messages that statistics convey to users will then clearly relate to each other, or at least will not contradict each other. The coherence between statistics is orientated towards the comparison of different statistics, which are generally produced in different way and for different primary uses (Eurostat 2000, S. 3).
\end{abstract}

Das Kriterium der Kohärenz ist in seiner Bedeutung für die verschiedenen Survey-Welten vergleichbar zum vorher diskutierten Kriterium der Vergleichbarkeit. Kohärenz macht wiederum keinen Sinn für die Beratungswelt, bzw. stellt eine Kontrastfolie für diese Welt dar, da hier spezifische, unvergleichbare Probleme durch eine beratende Survey-Firma gelöst werden sollen. In der Dienstleistungswelt hingegen muss die Kohärenz bei Bedarf durch die auftraggebende Organisation selbst hergestellt werden. In der Informationswelt ist die Kohärenz von Daten ein zentrales Kriterium, da erst die einheitliche Begriffsdefinition, Klassifikation und Methodik eine Vergleichbarkeit von unterschiedlichen Erhebungen ermöglicht. Kohärenz in der akademischen Welt ist wiederum ein Kriterium, welches die Vergleichbarkeit und damit die Kritisierbarkeit von bestehenden Erhebungen ermöglichen soll.

\section{Vollständigkeit (Completeness)}

Domains for which statistics are available should reflect the needs and priorities expressed by the users of the European Statistical System (Eurostat 2000 S. 3).

Das Kriterium der Vollständigkeit bezeichnet in der Beratungswelt die Eignung des Umfangs von Befragungen für die Erarbeitung einer Empfehlung. Da die Vollständigkeit durch den Berater selbst gesteuert wird, ist die Vollständigkeit im Hinblick auf die Daten kein sinnvolles Kriterium für diese Welt. Erst 
im Hinblick auf die Vollständigkeit der Empfehlungen wird es zu einem sinnvollen Evaluationskriterium für den Klienten. In der Dienstleistungswelt ist dieses Kriterium ebenfalls nur bedingt sinnvoll, da die Vollständigkeit der Daten durch den Kunden selbst gesteuert wird und dieser folglich selbst zuständig ist für die Erreichung dieser Qualität von Befragungen. Einen höheren Stellenwert bekommt dieses Kriterium wiederum bei dreiteiligen Value-Chains, wenn der Kunde der Survey-Firma also lediglich als Intermediär für interne Auftraggeber auftritt. In der Informationswelt stellt die Vollständigkeit ein zentrales Kriterium dar, da durch die generische Eigenheit der Datenproduktion keine direkte Verbindung zu den Datennutzenden besteht und die Vollständigkeit der Daten für die Nutzung eine aktive Vermittlung durch die Datenproduzenten notwendig macht. In der akademischen Welt schlussendlich bezieht sich das Kriterium der Vollständigkeit auf das Potential von Daten für die Beantwortung einer Forschungsfrage. Dies ist in dreiteiligen Value-Chains zentral, da hier die Datenproduktion nicht selbst durch Datenauswerter gesteuert wird. Die Vollständigkeit der Daten ist in einem solchen Fall jedoch nur schwierig durch Datenproduzenten zu steuern, da die Verwendung der Daten nur schwierig absehbar ist.

\section{Branchenkompetenz und persönliche Chemie}

- Problemverständnis, das in der Offerte zum Ausdruck kommt [...]

- Effektivität/Problemlösungskapazität der vorgeschlagenen Methode(n) [...]

- Qualifikation der Projektleiterin/persönlicher Eindruck

- Branchenkompetenz

- «Chemie» zwischen Projektverantwortlichem auf Auftraggeber-Seite und Projektleiterin des Instituts (Fankhauser und Wälty 2011, S. 115).

Das Lehrbuch zur Marktforschung von Fankhauser und Wälty bezeichnet verschiedene Qualitätskriterien für die Zusammenarbeit zwischen Survey-Firmen und Auftraggebern. In den folgenden Ausführungen werden zwei dieser Qualitätskriterien ausführlicher diskutiert, welche den survey-weltlichen Hintergrund der durch Fankhauser und Wälty definierten Qualitätskriterien besonders deutlich zum Ausdruck bringen. Im Hinblick auf das Kriterium der Branchenkompetenz zeigt sich ein zentraler Unterschied zwischen den generischen und den gewidmeten Welten. In den gewidmeten Welten stellt dies ein wichtiges Qualitätskriterium dar, jedoch in unterschiedlicher Art und Weise zu der von Fankhauser und Wälty intendierten Weise. In der Beratungswelt stellt die Branchenkompetenz die notwendige Grundlage für die inhaltliche beratende Tätigkeit in dieser Welt dar. Inhaltlich meint dabei, dass in dieser Welt nicht nur eine formale Beratung im Hinblick auf den Einsatz von methodischen 
Instrumenten stattfindet, sondern darüber hinaus auch ein Bezug auf die mittels der methodischen Instrumente zu lösenden Probleme geleistet wird. Der Bezug auf diese Problematik, welche durch die Befragung gelöst werden soll, bedingt der Branchenkompetenz und zusätzlich der Einarbeitung in die spezifische Problematik. In der Dienstleistungswelt dagegen ist Branchenkompetenz notwendig, um ein für eine Branche passendes Angebot erstellen zu können. Branchenkompetenz muss dadurch folglich nicht auf Projektleiterstufe wie in der Beratungswelt verfügbar sein. Es ist ausreichend, wenn die Verantwortlichen für die Angebotserstellung über Branchenkompetenz verfügen.

Ein weiteres von Fankhauser und Wälty bezeichnetes Qualitätskriterium für die Zusammenarbeit stellt die „,Chemie“ zwischen Projektleitern auf Auftraggeber-Seite und Projektleitern des Instituts“ dar (Fankhauser und Wälty 2011, S. 115). Hierbei zeigt sich, wie beim Kriterium der Branchenkompetenz, ein grundlegender Unterschied zwischen den generischen und den gewidmeten Survey-Welten. Dadurch, dass die generischen Survey-Welten einen vollständig vordefinierten Auftrag an die Survey-Firmen herantragen, ist die persönliche Chemie, d. h. das zwischenmenschliche Verhältnis bei der Projektzusammenarbeit, kein Kriterium für eine gelingende Zusammenarbeit. Die persönliche Chemie ist deswegen in der Informationswelt und in der akademischen Welt lediglich ein Kriterium für die zwischenmenschliche Sympathie während der Projektlaufzeit und damit der Annehmlichkeit der Zusammenarbeit. Anders sieht dies in den gewidmeten Welten aus. Insbesondere in der Beratungswelt stellt die persönliche Chemie zwischen Survey-Firma und Klient die Voraussetzung für eine gelingende Produktion von Empfehlungen dar, da es in dieser Welt der gelingende Austauschprozess zwischen beiden Parteien ist, welcher die schlussendliche Qualität der Wissensproduktion bestimmt. Die persönliche Chemie hat damit nicht lediglich Einfluss auf die Annehmlichkeit der Zusammenarbeit, sondern stellt darüber hinaus die Bedingung für einen gelingenden Survey-Prozess in dieser Welt dar. Die Bedeutung der persönlichen Chemie kommt in der Dienstleistungswelt hingegen nicht an die der Beratungswelt heran. Aber auch hier stellt aus Kundensicht die persönliche Chemie ein Qualitätskriterium der Zusammenarbeit dar, da dadurch allenfalls eine höhere Bereitschaft aufseiten der Survey-Firma für die Gewährung einer Flexibilität bei der Auftragsbearbeitung erreicht werden kann.

\section{Vertrauen}

Zaltman und Moorman (1988); Moorman et al (1993) weisen auf die zentrale Rolle von Vertrauen für geschäftliche Beziehungen zwischen Auftraggebenden und Survey-Firmen hin und erwähnen verschiedene Eigenschaften 


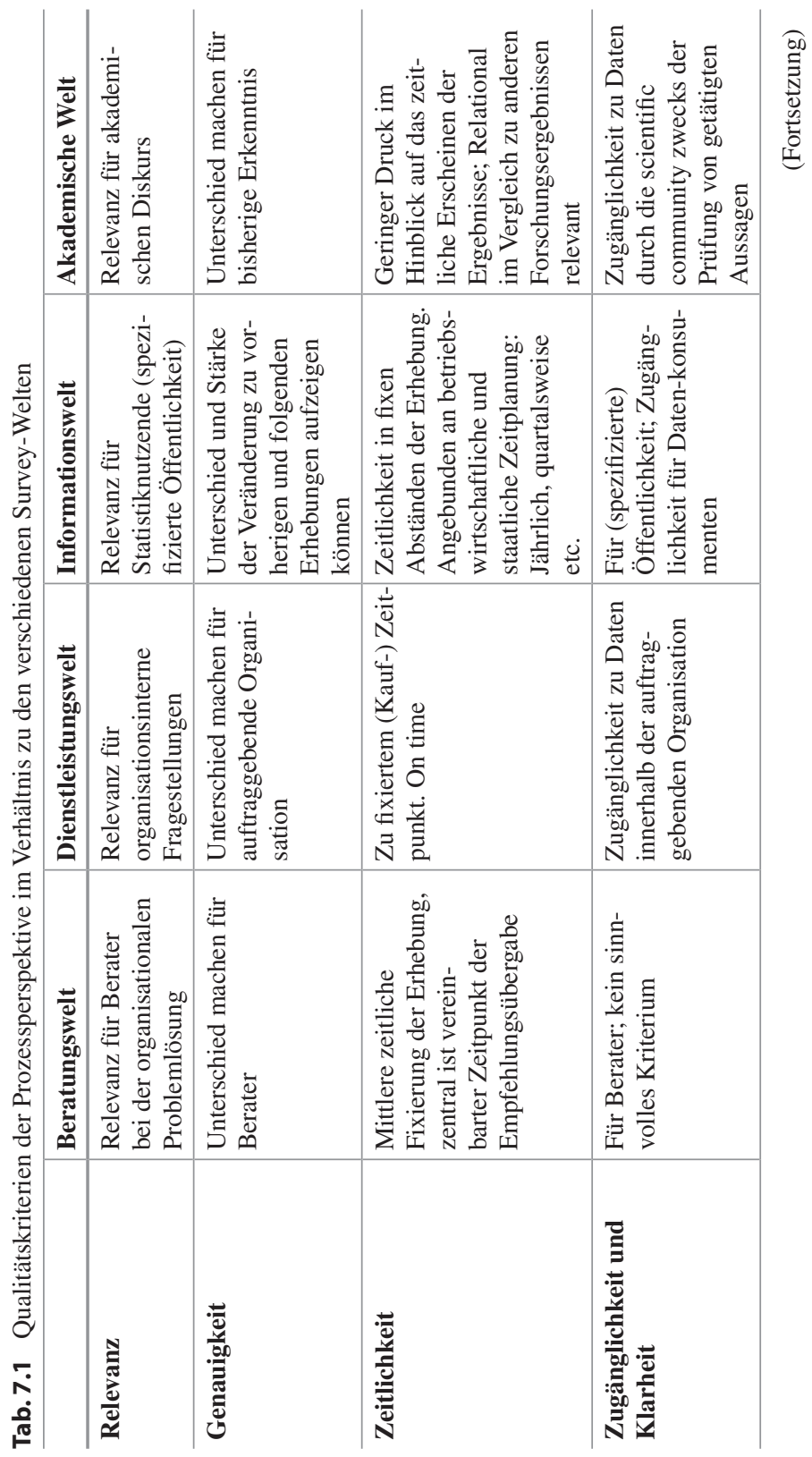




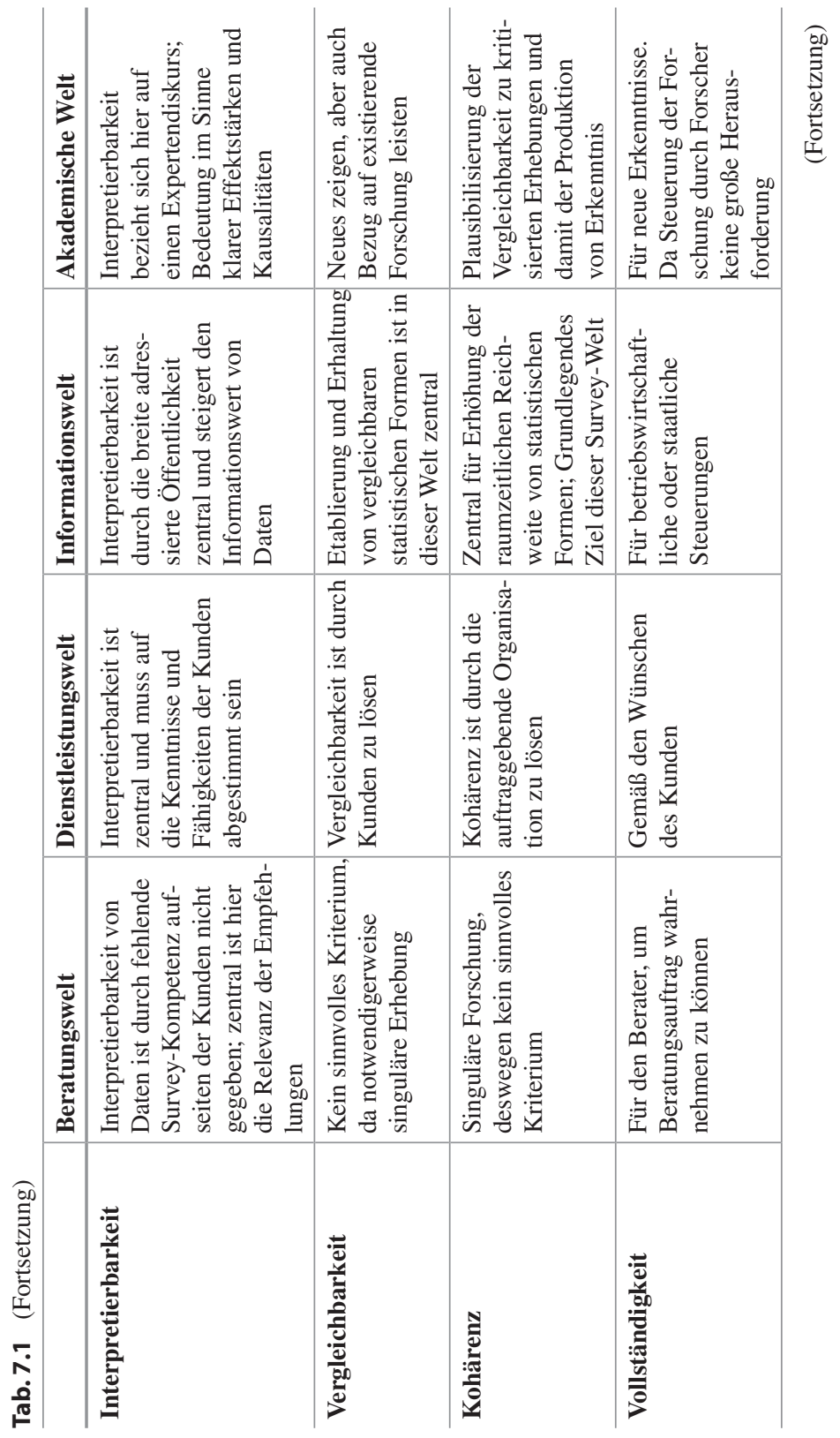




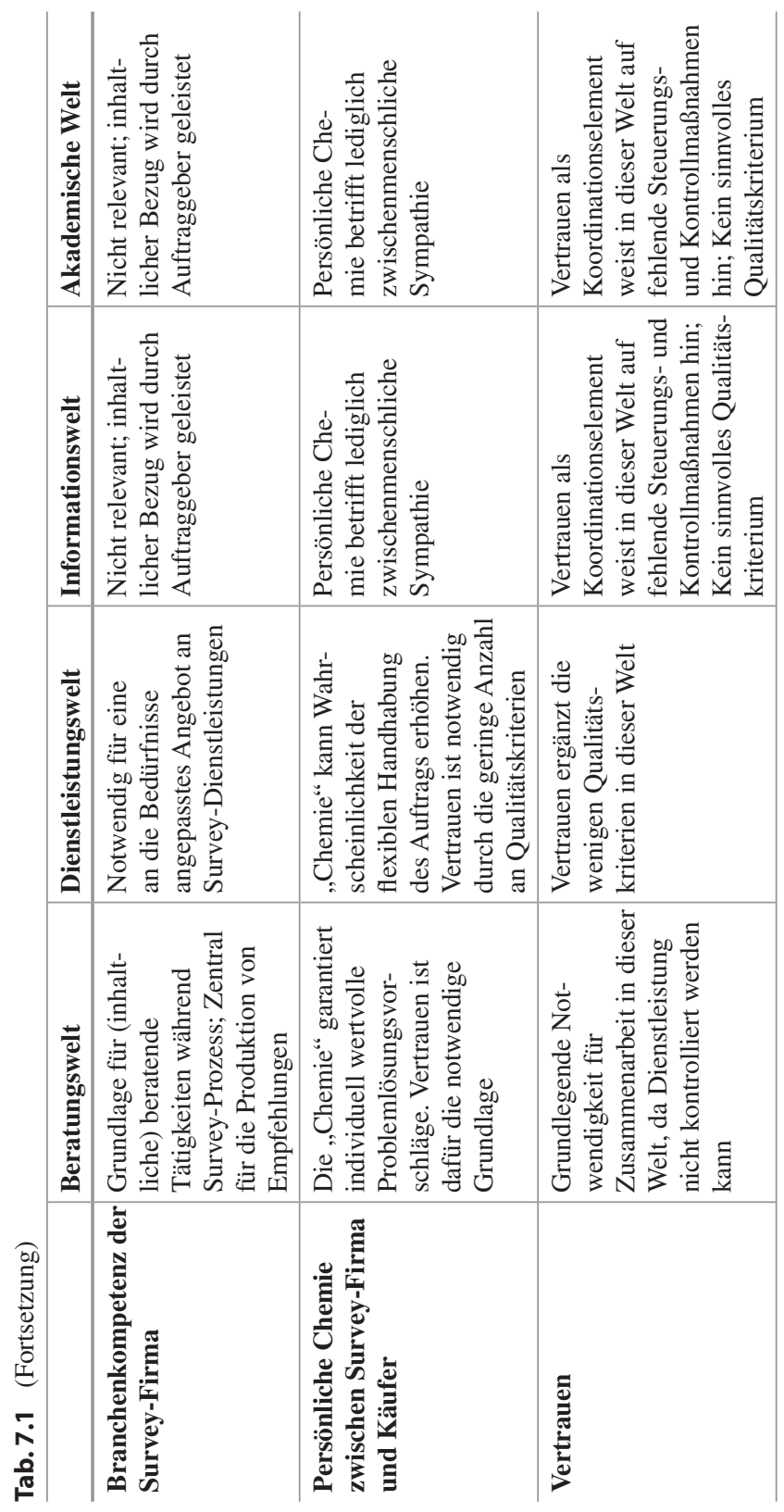


der Survey-Projektleitung, welche Vertrauen generieren. Sie kommen dabei zum Schluss, dass der persönlichen Beziehung in der Herstellung von Vertrauen das größte Gewicht bei den vertrauensgenerierenden Faktoren zukommt (Moorman et al. 1993). Vertrauen als Qualitätskriterium ist für die Beratungswelt am relevantesten. Da in dieser Survey-Welt die Klienten nicht das notwendige Knowhow haben, um die Qualität des Surveys einschätzen zu können, ist Vertrauen das wichtigste Qualitätskriterium für die Zusammenarbeit in dieser Survey-Welt. Eine mittlere Relevanz nimmt Vertrauen in der Dienstleistungswelt ein. Denn einerseits sind die Leistungen der Survey-Firmen beim Kauf festgelegt. Bedingt durch die geringe Anzahl an Qualitätskriterien in dieser Welt besteht jedoch auch nach der Festlegung der verschiedenen Kriterien noch ein großer Spielraum aufseiten der Survey-Firma bei der Bearbeitung des Auftrags. Im Gegensatz dazu spielt Vertrauen in der Informationswelt und in der akademischen Welt eine untergeordnete Rolle. Einerseits existiert aufseiten der Auftraggeber ein hohes SurveyKnow-how. Andererseits werden in diesen Welten umfassende Steuerungs- und Kontrollmaßnahmen eingesetzt, um die Erfüllung der survey-welt-spezifischen Qualitätskonzepte umsetzen zu können. Der Rückgriff auf Vertrauen als Koordinationsmechanismus verweist in diesen Welten auf fehlende Steuerungsund Kontrollmaßnahmen von Seiten der Auftraggeber.

In Tab. 7.1 sind die Relationen zwischen den erweiterten Qualitätskriterien der Prozessperspektive und den verschiedenen Survey-Welten tabellarisch dargestellt.

In diesem Kapitel wurden erweiterte Qualitätskriterien für die SurveyForschung im Hinblick auf ihre survey-weltliche Bedeutung und Anwendbarkeit diskutiert. Ziel war es dabei, die fehlende survey-weltliche Neutralität der erweiterten Qualitätskriterien darzustellen. Wie in Abschn. 2.4 argumentiert wurde, lassen sich die erweiterten Qualitätskriterien einer Prozessperspektive zurechnen, da hier nicht lediglich die Datenqualität, sondern darüber hinaus die Anwendungs- wie auch die Produktionssituation mit in die Konzeption von Survey-Qualität einbezogen wird. Bei der Diskussion der verschiedenen Qualitätskriterien wurde deutlich, dass sich die erweiterten Qualitätskriterien aus der „Marktforschung“ stärker auf die Produktions-, denn auf die Anwendungssituation konzentrieren. Die Trennung in unterschiedliche Aspekte der Survey-Qualität würde hier nahelegen, dass damit lediglich unterschiedliche Etappen im Survey-Produktionsprozess abgedeckt werden (Bachleitner et al. 2010, S. $151 \mathrm{ff}$.). Aus der Perspektive der Survey-Welten wird hingegen deutlich, dass mit dem unterschiedlichen Qualitätsfokus auch unterschiedliche Produktionslogiken von Surveys reflektiert werden. In der Beratungswelt beispielsweise ist der Prozess des Austauschs zwischen Berater und Klient eine zentrale Station, in welcher die Survey-Welten-spezifische Qualität der 
Empfehlung produziert wird. In den generischen Welten hingegen trägt dieser Austausch nicht gestaltend zur angestrebten survey-weltlichen Qualität bei, sondern stellt eher eine Problemsituation dar, da hier das vorgängig definierte Forschungsdesign durch das „Nadelöhr“ der interorganisationalen Kommunikation vermittelt werden muss. Der starke Fokus der erweiterten Qualitätskriterien auf den Prozess der Survey-Erstellung trägt folglich exakt diesem Umstand des unterschiedlichen Stellenwerts des organisationalen Austauschs in den verschiedenen Survey-Welten Rechnung. Hingegen reflektieren die erweiterten Qualitätskriterien des Quality-Movements (Desrosières 2009a) primär die Problematik der generischen Welt der Informationswelt, die generische Informationsproduktion an den tatsächlichen Bedürfnissen der Statistiknutzenden auszurichten. Diese starke Distribution der Informationsproduktion über verschiedene Stellen hinweg ist dabei gerade typisch für die generischen Welten, in den gewidmeten Welten hingegen viel weniger der Fall. Die Orientierung an den Bedürfnissen der Statistiknutzenden ist beispielsweise in der Beratungswelt kein sinnvolles Qualitätskriterium, da die Klienten gleichzeitig auch die Adressaten der Survey-Produktion darstellen. Der Adressat der SurveyProduktion ist in dieser Welt mit dem Auftraggeber gleichgesetzt. Deutlich wird damit, dass die verschiedenen analysierten erweiterten Qualitätskriterien keineswegs survey-weltlich neutrale Orientierungspunkte für die Survey-Produktion darstellen. Insbesondere auch der Fokus auf die Anwendungssituation als Erweiterung der Qualitätsperspektive, wie dies durch das Quality-Movement vorgeschlagen wird (Desrosières 2009a; Eurostat 2000; Statistics Canada 2009) und beispielsweise durch Biemer und Lyberg aufgenommen wurde (2008), stellt keinen für die verschiedenen Survey-Welten universell gangbaren Weg einer umfassenden Qualitätsperspektive dar. Zentral bei der Definition und der Anwendung von erweiterten Qualitätskriterien ist folglich der Miteinbezug von survey-weltlichen Prozess- und Produktionslogiken.

\subsection{Ein neues Verständnis von methodischen Problemen in Survey-Projekten}

Im vorherigen Kapitel wurde die kontingente Relevanz verschiedener Qualitätskriterien für die verschiedenen Survey-Welten dargestellt. Es wurde aufgezeigt, dass in der Survey-Methodologie etablierte Qualitätskriterien aus der Perspektive der verschiedenen Survey-Welten entweder eine unterschiedliche Spezifizierung erlangen oder dann nur eine selektive Gültigkeit haben. Im Folgenden wird eine survey-weltliche Perspektive auf Methodenprobleme in Surveys entworfen, 
in welcher Konflikte zwischen verschiedenen Wissensformaten im Fokus stehen und deren Einfluss auf die survey-pragmatischen Elemente der Methodik und des Survey-Managements. Es findet folglich eine Generalisierung von in den Fallanalysen identifizierten und analysierten survey-weltlichen Koordinationsproblemen statt. Das Ziel liegt dabei nicht in der Erstellung neuer Qualitätskriterien, welche die verschiedenen Survey-Welten abdecken, sondern vielmehr in der Darstellung der Konsequenzen eines fehlenden survey-weltlichen methodischen Holismus. Das Konzept des methodischen Holismus geht von einer gegenseitigen Bedingung zwischen Theorie, Methodologie und Methode aus (Diaz-Bone 2010c, S. 183 ff.). Der methodische Holismus bezeichnet eine theoriegesteuerte Methodik. Dies bedeutet, dass empirische Forschung und die dafür verwendeten methodischen Instrumente mit der theoretischen Fundierung der Forschung kompatibel sein müssen. Das empirische Forschen ist entsprechend nicht eine separate, von der Theoriearbeit losgelöste Etappe des Forschungsprozesses, sondern soll eine Realisierung der theoretischen Annahmen darstellen. Damit wird ein Qualitätsmaßstab für Forschung etabliert, welcher das Verhältnis zwischen der theoretischen Grundlegung und der methodischen Umsetzung ins Zentrum der Forschungsorganisation stellt. Beispiele der Umsetzung eines methodischen Holismus finden sich in der bourdieuschen Soziologie (Bourdieu 1987; Bourdieu et al. 1991, S. 46 ff.) und in den Arbeiten von Barney Glaser und Anselm Strauss im Hinblick auf die Grounded Theory (Glaser und Strauss 1967; Strauss 1998). ${ }^{28}$

Das Konzept des methodischen Holismus kann auf Survey-Welten bezogen werden. Wie in den beiden Fallrekonstruktionen gezeigt wurde, sind uneinheitliche Bezüge auf Survey-Welten eine Ursache für methodische Probleme in Survey-Projekten. Beispiele hierfür sind die Diskussion um die Kategorie „Staatsangehörigkeit" in Fall (A) (vgl. Abschn. 6.1.3) und die Diskussion um das Storytelling des Fragebogens in Fall (B) (vgl. Abschn. 6.2.4). In beiden genannten Beispielen wurde deutlich, dass eine fehlende survey-weltliche Abstimmung direkte methodische Konsequenzen hätte. Survey-weltliche Koordinationsprobleme können zwei Ursachen haben. Einerseits kann eine Differenz im Hinblick auf den survey-weltlichen Bezug zwischen Auftraggeber und Auftragnehmer zu einem survey-weltlichen Koordinationsproblem führen.

\footnotetext{
${ }^{28}$ Die bourdieusche Soziologie und deren methodischer Holismus greifen dabei auf Vorarbeiten des französischen Epistemologen Gaston Bachelard zurück. Dieser schreibt: „Man muß nachdenken, um zu messen, und nicht messen, um nachzudenken“ (1987, S. 309).
} 


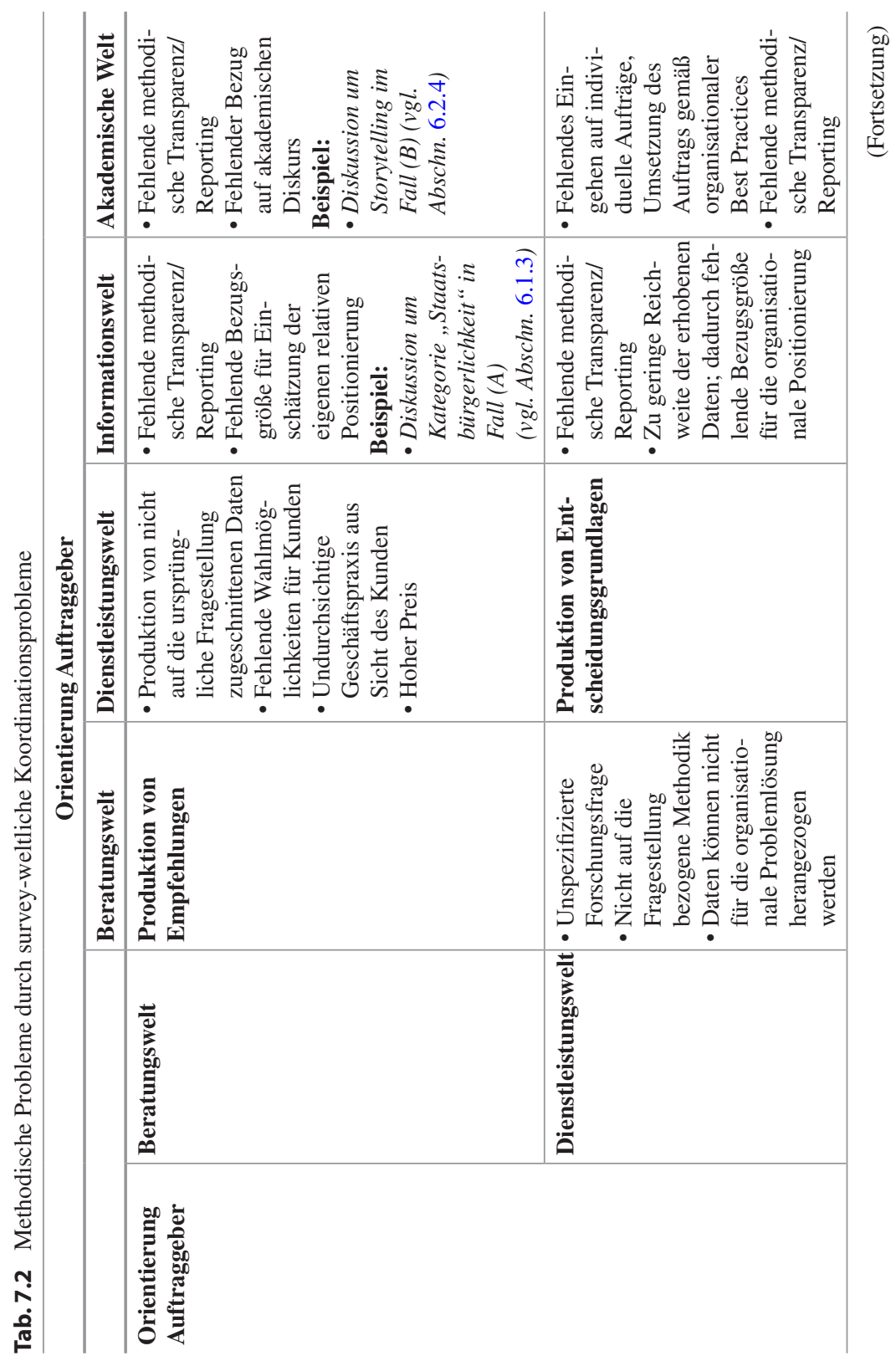




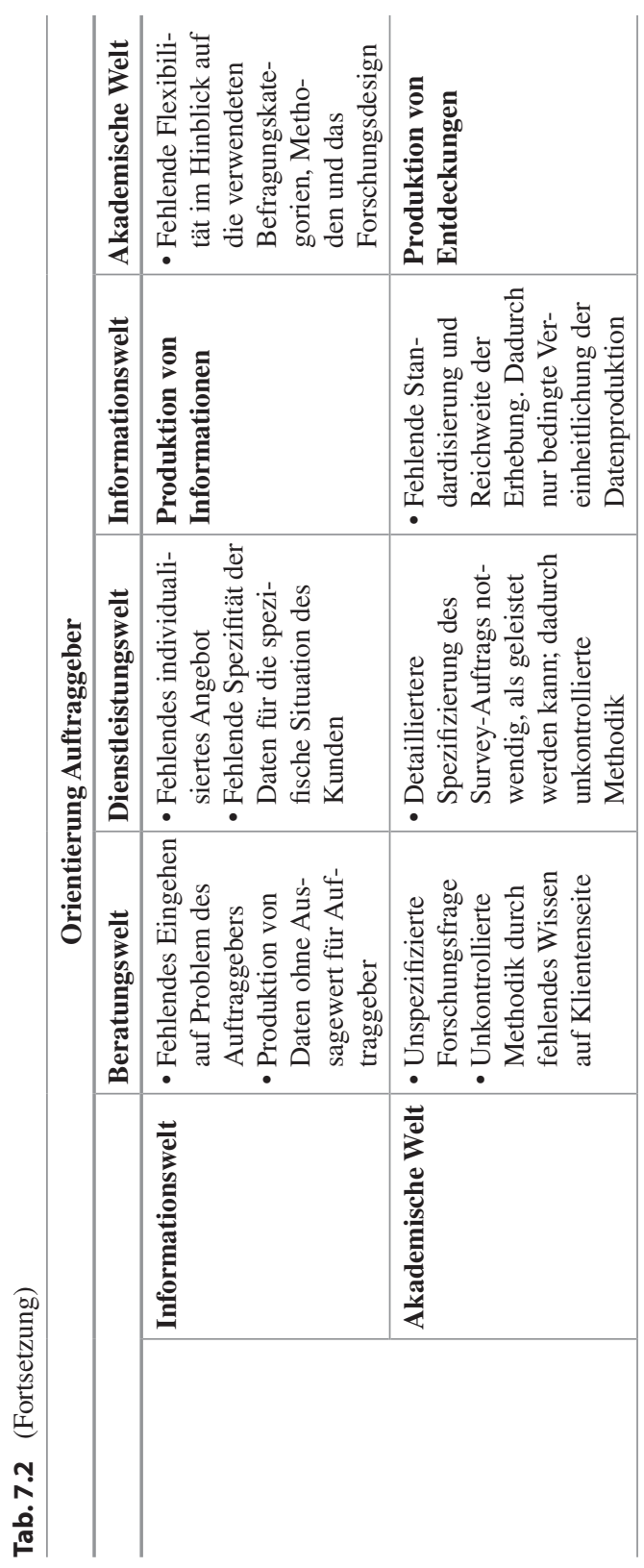


Die Problematik liegt in diesem Fall in einem uneinheitlichen Bezug und damit einer fehlenden Koordination von survey-weltlichen Konventionen im Hinblick auf ein Survey-Projekt. ${ }^{29}$ Eine zweite Ursache von survey-weltlichen Koordinationsproblemen liegt im unvollständigen Bezug auf survey-weltliche Konventionen. Dies ist beispielsweise dann der Fall, wenn sich Käufer an Angeboten der Dienstleistungswelt orientieren, jedoch nicht über die dafür notwendige Kundenkompetenz verfügen. Ein anderes Beispiel für diese zweite Ursache von survey-weltlichen Koordinationsproblemen stellt der Fall dar, in welchem Käufer als Auftraggeber die verschiedenen Elemente eines Survey-Projekts umfangreich selber steuern wollen, jedoch nicht über die notwendigen Kenntnisse der verschiedenen Steuerungsmöglichkeiten verfügen. In beiden Beispielen ist ein Kompromiss mit der Beratungswelt notwendig, um eine erfolgreiche Auswahl, bzw. eine erfolgreiche Steuerung, gewährleisten zu können.

Koordinationsprobleme können im Hinblick auf sämtliche beschriebenen Konventionen der Survey-Welten entstehen (vgl. hierzu Abschn. 5.6). Die Konsequenzen von surveyweltlichen Koordinationsproblemen sind vielfältig. Sie betreffen nicht lediglich die verschiedenen Wissensformate der Survey-Welten. In Abschn. 2.5.5 wurde das Konzept der Survey-Pragmatik eingeführt. Damit sollte eine nicht-reduktionistische Perspektive auf die Koordination von Survey-Projekten eingeführt werden, welche einen Fokus auf die Produktion von unterschiedlichen Wissensformaten legt, diese jedoch nicht als alleinigen Gegenstand der Koordination betrachtet. Entsprechend haben survey-weltliche Koordinationsprobleme nicht lediglich Probleme bezüglich des Wissensformats zur Folge, sondern wirken sich auch auf die beiden anderen Dimensionen der Survey-Pragmatik, die Methodik und das Survey-Management von SurveyProjekten aus. In Tab. 7.2 werden verschiedene survey-weltliche Koordinationsprobleme idealtypisch dargestellt. Dargestellt werden Koordinationsprobleme, welche sich aus einer unterschiedlichen survey-weltlichen Orientierung zwischen Auftraggeber und Auftragnehmer ergeben. Die dargestellten Koordinationsprobleme sind idealtypisch dargestellt. Nichtsdestotrotz stellen einige dieser Koordinationsprobleme konkrete in den Daten identifizierbare Koordinationsprobleme dar.

Der Ursprung von survey-weltlichen Koordinationsproblemen kann nicht einfach der Auftraggeber- oder der Auftragnehmerseite zugerechnet werden, was durch die unterschiedlichen Koordinations- und Verantwortungslogiken

\footnotetext{
${ }^{29}$ Was Kompromisse nicht ausschließt, da diese in gegenseitiger Abstimmung etabliert werden können.
} 
der verschiedenen Survey-Welten begründet ist. Wie in Fall (A) deutlich wurde, kommt beispielsweise in der Beratungswelt ${ }^{30}$ dem Berater die Rolle zu, die Kompetenzen des Auftragnehmers im Hinblick auf die verschiedenen Dimensionen der Survey-Pragmatik zu erkennen und in die Planung miteinzubeziehen (vgl. Abschn. 6.1). Diese Verantwortung - und damit auch die Möglichkeit zur Governance des Survey-Projekts - dreht sich in Fall (B) um, da es in diesem Fall der Auftraggeber ist, welcher als Treiber der Survey-Pragmatik fungiert. Die Verortung der beschriebenen Koordinationsprobleme gestaltet sich folglich abhängig von der Survey-Welt sehr unterschiedlich.

Die Entstehung von survey-weltlichen Koordinationsproblemen ist jedoch nicht immer nur Missverständnissen oder fehlenden Kompetenzen auf Auftraggeber- oder Auftragnehmerseite zuzurechnen, sondern kann auch mit den Eigenheiten der verschiedenen Survey-Welten zusammenhängen. In Abschn. 7.1 wurde die Marktproblematik der Beratungswelt dargestellt. Diese liegt darin, dass der Produktcharakter der angebotenen Dienstleistung sehr gering ist, da das Produkt erst durch den Austausch mit dem Klienten entsteht. Zusätzlich sind die Preise im Vergleich zur Dienstleistungswelt geringer, da Economies of Scale weniger stark zum Tragen kommen durch die geringe Standardisierung der Survey-Produktion. Durch die starke Prozessualität der Survey-Produktion ist es zudem erst sehr spät im Survey-Prozess möglich, die eigentliche Qualität der Befragung abschätzen zu können. In einem direkten Vergleich vor dem Beginn eines Survey-Projekts ist die Dienstleistungswelt dadurch oft konkurrenzfähiger, insbesondere im Hinblick auf die preisliche Konkurrenzfähigkeit. Diese Problematik wird im folgenden Zitat durch einen Projektleiter bei einer Survey-Firma thematisiert:

[...] also ich hatte letzte Woche mit einer vom [staatliche Stelle] Kontakt, die äh Kampagnenevaluation wollte, mit der hatte ich auch eben telefoniert und wir haben auch kurz am Telefon mögliche Fragebogenaufbau und Einbindung ihrer Bilder und so besprochen und ihr dann die Offerte geschickt und dann kam dann eben zurück, ähm, und da merkst du dann, dass sie das eigentlich schon noch wichtig, weil es tue ihr extrem leid, aber sie hätte einen anderen Anbieter nehmen müssen, der für äh, der über einen Drittel günstiger war. Obwohl sie [...] meine Offerte und meine Inputs mit Abstand am besten gefunden hätte. [...] Und da spielt dann, eben, klar eben sie hat schon auch gemeint, es tue ihr leid, weil eben von mir schon sehr viel Feedback und Inputs gekommen sei, was sie jetzt bei den anderen jetzt halt noch machen muss. [...] Aber, ähm, sie musste dann halt, sie hat die drei Offerten

\footnotetext{
${ }^{30}$ In deren Kompromissschliessung im bezeichneten Survey-Projekt mit anderen SurveyWelten.
} 
gekriegt und musste mit denen dann beim Chef anfragen, ob sie das Geld kriegt [...]. Und der sieht dann natürlich einfach drei Preise. Und dann hast du gerade als [staatliche Stelle], die eben ein bisschen im Moment äh, Schiss haben, dass sie jedes Mal, wenn sie was nicht beim Günstigsten vergeben, weil sie von der Finanzaufsicht gelyncht werden. Es ist natürlich immer, das sind einfach, ja, das sind einfach sichtbare und gut vergleichbare Werte, während dem du eben inhaltliche Qualität halt eben nicht so quantifizieren kannst.

Deutlich wird in diesem Zitat, dass sich die Leitungsperson auf Qualitätskriterien der Dienstleistungswelt bezieht, währenddem die mit dem Survey-Projekt beauftragte Person das Survey-Projekt mit dem stärker beratungsweltlichen Charakter präferiert. ${ }^{31}$ Deutlich werden aus diesem Fallbeispiel zwei Punkte: Einerseits wird wiederum die begrenzte raumzeitliche Reichweite der beratungsweltlichen Qualität deutlich. Erst durch das spezifische Eingehen des Projektleiters auf die Bedürfnisse der für den Survey beauftragten Person wird für diese die Qualität der Planung des Projektleiters deutlich. Die Kommunikation gegenüber dem Vorgesetzten gestaltet sich dagegen bereits schwieriger, da sich die Kommunikation der Qualität nicht auf allgemeine Marktstandards wie in der Dienstleistungswelt stützen kann. Zweitens wird aber auch deutlich, dass die Finanzierungsvorgaben des Bundes die Beratungswelt systematisch auszuschließen scheinen, da der Preis als primäres Evaluationskriterium herangezogen wird. Nicht beachtet wird mit dieser Vorgabe, dass der Preis als primäres Entscheidungskriterium nur dann Sinn macht, wenn Klarheit und Einigkeit über die zu erreichende Qualität der Befragung herrscht (Favereau 1989a). Dies ist jedoch offensichtlich nicht der Fall, da die spezifische Qualität erst durch die Lektüre der verschiedenen Offerten für die zuständige Person sichtbar wird. Das auf den Preis fokussierte Entscheidungskriterium setzt eine Vergleichbarkeit der Qualität zwischen den verschiedenen Offerten voraus, welche jedoch offensichtlich nicht gegeben ist. Dadurch wird eine Hegemonialisierung der Dienstleistungswelt deutlich, welche die beratungsweltliche Koordinationsgrundlage systematisch ausschließt, da diese preislich unmöglich mit der Dienstleistungswelt konkurrenzieren kann. ${ }^{32}$

\footnotetext{
${ }^{31}$ Wobei hier auch beide Positionen auf einem Kompromiss zwischen der Beratungswelt und der Dienstleistungswelt fußen, welcher graduell unterschiedlich ausfällt.

${ }^{32}$ Mit Thévenot könnte man hier von einem „Governing by Standards“ sprechen (Thévenot 2009b). Denn auch hier wird von einer auf „Objektivität“ von Qualität basierenden Regulation ausgegangen, welche eine Standardisierung erst ermöglicht. Insbesondere der Bezug auf das Regime des Vertrauten als genuinem Bestandteil der Beratungswelt wird damit verunmöglicht, da es sich der Standardisierbarkeit widersetzt.
} 
Obwohl der Koordinationsrahmen der restlichen Offerten nicht geklärt werden kann, werden doch potentielle survey-weltliche Koordinationsprobleme sichtbar. Idealtypisch sind hier eine unspezifizierte Forschungsfrage und eine nicht auf die Fragestellung bezogene Methodik zu nennen, sodass die Daten schlussendlich nicht für die angestrebte Entscheidungsfindung herangezogen werden können.

Open Access Dieses Kapitel wird unter der Creative Commons Namensnennung 4.0 International Lizenz (http://creativecommons.org/licenses/by/4.0/deed.de) veröffentlicht, welche die Nutzung, Vervielfältigung, Bearbeitung, Verbreitung und Wiedergabe in jeglichem Medium und Format erlaubt, sofern Sie den/die ursprünglichen Autor(en) und die Quelle ordnungsgemäß nennen, einen Link zur Creative Commons Lizenz beifügen und angeben, ob Änderungen vorgenommen wurden.

Die in diesem Kapitel enthaltenen Bilder und sonstiges Drittmaterial unterliegen ebenfalls der genannten Creative Commons Lizenz, sofern sich aus der Abbildungslegende nichts anderes ergibt. Sofern das betreffende Material nicht unter der genannten Creative Commons Lizenz steht und die betreffende Handlung nicht nach gesetzlichen Vorschriften erlaubt ist, ist für die oben aufgeführten Weiterverwendungen des Materials die Einwilligung des jeweiligen Rechteinhabers einzuholen. 